TRANSACTIONS OF THE

AMERICAN MATHEMATICAL SOCIETY

Volume 355, Number 6, Pages 2523-2543

S 0002-9947(03)03261-6

Article electronically published on January 30, 2003

\title{
CONSTRUCTION OF $t$-STRUCTURES AND EQUIVALENCES OF DERIVED CATEGORIES
}

\author{
LEOVIGILDO ALONSO TARRÍO, ANA JEREMÍAS LÓPEZ, \\ AND MARÍA JOSÉ SOUTO SALORIO
}

\begin{abstract}
We associate a $t$-structure to a family of objects in $\mathbf{D}(\mathcal{A})$, the derived category of a Grothendieck category $\mathcal{A}$. Using general results on $t$ structures, we give a new proof of Rickard's theorem on equivalence of bounded derived categories of modules. Also, we extend this result to bounded derived categories of quasi-coherent sheaves on separated divisorial schemes obtaining, in particular, Beĭlinson's equivalences.
\end{abstract}

\section{INTRODUCTION}

One important observation in modern homological algebra is that sometimes equivalences of derived categories do not come from equivalences of the initial abelian categories. A remarkable example of this situation is that of complexes of holonomic regular $\mathcal{D}$-modules and complexes of locally constructible finite type sheaves of vector spaces over an analytic variety. In fact, to a single regular holonomic $\mathcal{D}$-module there corresponds a full complex of constructible sheaves which are called "perverse sheaves". In turn, these sheaves are connected with intersection cohomology that allows us to recover Poincaré duality for singular analytic spaces.

It is a crucial fact that a (bounded) derived category $\mathbf{D}^{\mathrm{b}}$ may contain, as full subcategories, abelian categories that are not isomorphic to the initial one but whose (bounded) derived category is isomorphic to $\mathbf{D}^{\mathrm{b}}$. These facts have been categorized by Beulinson, Bernstein, Deligne and Gabber in BBD through the concept of $t$-structures. A $t$-structure in a triangulated category $\mathbf{T}$ is given by functors which resemble the usual truncation functors in derived categories and provide as a by-product an abelian full subcategory $\mathcal{C}$ of $\mathbf{T}$, its heart, together with a homological functor on $\mathbf{T}$ with values in $\mathcal{C}$.

A derived category carries a natural $t$-structure but can also carry others. It is clear that the construction of a $t$-structure without recourse to an equivalence of derived categories is an important question. In fact, this is how perverse sheaves are transposed to the étale context where it is not possible to define them through an equivalence.

But, up to now, there was no general method available to construct $t$-structures, except for the glueing method in [BBD 1.4] whose main application is to certain categories of sheaves over a site. In this paper, we give such a general method for

Received by the editors May 14, 2002 and, in revised form, October 30, 2002.

2000 Mathematics Subject Classification. Primary 18E30; Secondary 14F05, 16D90.

The first two authors were partially supported by Spain's MCyT and E.U.'s FEDER research project BFM2001-3241, supplemented by Xunta de Galicia grant PGDIT 01PX120701PR. 
the derived category of a Grothendieck abelian category (i.e., an abelian category with a generator and exact direct limits). These categories arise often in representation theory and algebraic geometry. Our method parallels the fruitful method of localization used frequently in homotopy theory and algebra.

We believe our result is widely applicable. In this paper we begin to explore these possibilities. We show that the remarkable theorem of Rickard, that characterizes when two bounded derived categories of rings are equivalent, can be deduced from our construction and standard facts on $t$-structures. Moreover, we show that Rickard's result in $[\underline{R}$ can be extended to certain schemes. Precisely, the bounded derived category of quasi-coherent sheaves on a divisorial scheme is equivalent to the bounded derived categories of modules over a certain ring if there exists a special kind of "tilting complex" of sheaves. This generalizes Beĭlinson's equivalence in [B1] and [B2], in the sense that such an equivalence follows from this result.

Let us discuss now the contents of the paper. The first section recalls the basic notions of $t$-structures and highlights the observation by Keller and Vossieck that to give a $t$-structure $\left(\mathbf{T}^{\leq 0}, \mathbf{T}^{\geq 0}\right)$ it is enough to have the subcategory $\mathbf{T}^{\leq 0}$ and a right adjoint for the canonical inclusion functor $\mathbf{T}^{\leq 0} \subset \mathbf{T}$. This has led us to search for a proof in the spirit of Bousfield localization, using techniques similar to those in [AJS. We will need to work in the unbounded derived category because the existence of coproducts is essential for our arguments.

For a triangulated category $\mathbf{T}$, a suspended subcategory stable for coproducts is called a cocomplete pre-aisle. In the second section we prove that these subcategories are stable for homotopy direct limits. In the next section we prove that if a cocomplete pre-aisle is generated by a set of objects, then it is in fact an aisle, i.e., a subcategory similar to $\mathbf{T}^{\leq 0}$. As in the case of Bousfield localizations, we proceed in two steps. First, we deal with the case of derived categories of modules over a ring, and next we treat the general case using the derived version of the Gabriel-Popesco embedding.

In the fourth section, we investigate the important question of whether the $t$ structures previously defined restrict to the bounded derived category of modules over a ring. Restriction to the upper-bounded category is easy; however, for the lower-bounded case some extra work is needed. We obtain this restriction when the $t$-structure is generated by a compact object that also generates the derived category in the sense of triangulated categories.

In the fifth section we deal with the analogous question in the context of schemes. This section may be skipped if the reader is only interested in the ring case. Trying to extend the arguments that work for rings in this context, we need a condition on compatibility with tensor products in order to localize to open subsets. This condition led us to define rigid aisles for which we give in Proposition 5.1 a criterion useful in practice.

In the sixth section we see that the functor "real" defined in [BBD, Chap. III] induces an equivalence of categories between the bounded derived category of the heart of a $t$-structure and the bounded derived category of modules over a ring precisely when the $t$-structure is generated by a tilting object. The heart is equivalent to a category of rings in this case - recovering Rickard's equivalence theorem. The generalization to the bounded derived category of quasi-coherent sheaves gives a similar equivalence when the $t$-structure is rigid and generated by a tilting object, thus making it equivalent to a bounded derived category of modules over a ring. We 
explain how this result is related to Beilinson's description of the bounded derived category of coherent sheaves on projective space as a bounded derived category of modules over a finite-dimensional algebra.

Acknowledgment. We thank Amnon Neeman for discussions on these topics.

\section{Preliminaries on $t$-Structures}

Let us begin by fixing the notation and conventions. Let $\mathbf{T}$ be a triangulated category whose translation auto-equivalence is denoted by $(-)[1]$ and its iterates by $(-)[n]$, with $n \in \mathbb{Z}$. A t-structure on $\mathbf{T}$ in the sense of Bellinson, Bernstein, Deligne and Gabber ( $\mathrm{BBD}$, Définition 1.3.1]) is a couple of full subcategories $\left(\mathbf{T}^{\leq 0}, \mathbf{T}^{\geq 0}\right)$ such that, denoting $\mathbf{T}^{\leq n}:=\mathbf{T}^{\leq 0}[-n]$ and $\mathbf{T}^{\geq n}:=\mathbf{T}^{\geq 0}[-n]$, the following conditions hold:

(t1) For $X \in \mathbf{T}^{\leq 0}$ and $Y \in \mathbf{T}^{\geq 1}$, $\operatorname{Hom}_{\mathbf{T}}(X, Y)=0$.

(t2) $\mathbf{T}^{\leq 0} \subset \mathbf{T}^{\leq 1}$ and $\mathbf{T}^{\geq 0} \supset \mathbf{T}^{\geq 1}$.

(t3) For each $X \in \mathbf{T}$ there is a distinguished triangle

$$
A \longrightarrow X \longrightarrow B \stackrel{+}{\longrightarrow}
$$

with $A \in \mathbf{T}^{\leq 0}$ and $B \in \mathbf{T}^{\geq 1}$.

The subcategory $\mathbf{T}^{\leq 0}$ is called the aisle of the $t$-structure, and $\mathbf{T}^{\geq 0}$ is called the co-aisle. As usual, for a subcategory $\mathcal{C} \subset \mathbf{T}$, we denote the associated orthogonal subcategories as $\mathcal{C}^{\perp}=\left\{Y \in \mathbf{T} / \operatorname{Hom}_{\mathbf{T}}(Z, Y)=0, \forall Z \in \mathcal{C}\right\}$ and ${ }^{\perp} \mathcal{C}=\{Z \in$ $\left.\mathbf{T} / \operatorname{Hom}_{\mathbf{T}}(Z, Y)=0, \forall Y \in \mathcal{C}\right\}$. Let us recall some immediate formal consequences of the definition.

Proposition 1.1. Let $\mathbf{T}$ be a triangulated category, $\left(\mathbf{T}^{\leq 0}, \mathbf{T}^{\geq 0}\right)$ a t-structure in $\mathbf{T}$, and $n \in \mathbb{Z}$. Then

(i) $\left(\mathbf{T}^{\leq 0}, \mathbf{T}^{\geq 1}\right)$ is a pair of orthogonal subcategories of $\mathbf{T}$, i.e., $\mathbf{T}^{\geq 1}=\mathbf{T}^{\leq 0^{\perp}}$ and $\mathbf{T}^{\leq 0}={ }^{\perp} \mathbf{T} \geq 1$.

(ii) The subcategories $\mathbf{T}^{\leq n}$ are stable for positive translations and the subcategories $\mathbf{T}^{\geq n}$ are stable for negative translations.

(iii) The canonical inclusion $\mathbf{T} \leq n \hookrightarrow \mathbf{T}$ has a right adjoint denoted $\tau \leq n$, and $\mathbf{T}^{\geq n} \hookrightarrow \mathbf{T}$ has a left adjoint denoted $\tau^{\geq n}$. Moreover, $X \in \mathbf{T}^{\leq n}$ if, and only if, $\tau^{\geq n+1}(X)=0$; similarly for $\mathbf{T}^{\geq n}$.

(iv) For $X$ in $\mathbf{T}$ there is a distinguished triangle

$$
\tau^{\leq 0} X \longrightarrow X \longrightarrow \tau^{\geq 1} X \stackrel{+}{\longrightarrow} .
$$

(v) The subcategories $\mathbf{T}^{\leq n}$ and $\mathbf{T}^{\geq n}$ are stable for extensions, i.e., given a distinguished triangle $X \rightarrow Y \rightarrow Z \stackrel{+}{\rightarrow}$, if $X$ and $Z$ belong to one of these categories, so does $Y$.

Proof. The statement ( $i$ ) follows easily from the axioms, and $(i i)$ is a restatement of (t2). The statements (iii) and (iv) are proved in [BBD, Prop. 1.3.3]. Finally, we sketch a proof of $(v)$ for $\mathbf{T}^{\leq n}$; the one for $\mathbf{T}^{\geq n}$ is dual. By translation, we can even restrict to $\mathbf{T}^{\leq 0}$. Apply $\operatorname{Hom}_{\mathbf{T}}\left(-, \tau^{\geq 1} Y\right)$ to the triangle and get the long exact sequence of abelian groups

$$
\cdots \rightarrow \operatorname{Hom}_{\mathbf{T}}\left(Z, \tau^{\geq 1} Y\right) \rightarrow \operatorname{Hom}_{\mathbf{T}}\left(Y, \tau^{\geq 1} Y\right) \rightarrow \operatorname{Hom}_{\mathbf{T}}\left(X, \tau^{\geq 1} Y\right) \rightarrow \ldots
$$


where $X$ and $Z$ belong to $\mathbf{T}^{\leq 0}={ }^{\perp} \mathbf{T}^{\geq 1}$. Therefore, both extreme homs are zero and by exactness

$$
\operatorname{Hom}_{\mathbf{T}}\left(\tau^{\geq 1} Y, \tau^{\geq 1} Y\right) \cong \operatorname{Hom}_{\mathbf{T}}\left(Y, \tau^{\geq 1} Y\right)=0,
$$

which means that $\tau^{\leq 0} Y=Y$, i.e., $Y \in \mathbf{T}^{\leq 0}$.

The interest of this notion lies in the fact that a $t$-structure $\left(\mathbf{T}^{\leq 0}, \mathbf{T}^{\geq 0}\right)$ in a triangulated category $\mathbf{T}$ provides a full abelian subcategory of $\mathbf{T}$, its heart, defined as $\mathcal{C}:=\mathbf{T}^{\leq 0} \cap \mathbf{T}^{\geq 0}$ and a homological functor, namely $H^{0}:=\tau^{\geq 0} \tau^{\leq 0}$, with values in $\mathcal{C}$; see $[\mathrm{BBD}$, Thm. 1.3.6]. One would like to know natural conditions that guarantee the existence of a $t$-structure in a triangulated category. We will show how to transpose homotopical techniques to get means of constructing $t$-structures.

A fundamental observation is to use the structure of the subcategories $\mathbf{T} \leq n$ and $\mathbf{T}^{\geq n}$. It is clear that, in general, they are not triangulated subcategories but they come close. In fact, each subcategory $\mathbf{T}^{\leq n}$ has the structure of suspended category in the sense of Keller and Vossieck [KV1]. Let us recall this definition.

An additive category $\mathcal{U}$ is suspended if and only if it is graded by an additive translation functor $T$ (sometimes called shifting) and there is a class of diagrams of the form $X \rightarrow Y \rightarrow Z \rightarrow T X$ (often denoted simply $X \rightarrow Y \rightarrow Z \stackrel{+}{\rightarrow}$ ) called distinguished triangles such that the following axioms, which are analogous to those for triangulated categories in Verdier's work [V, p. 266] hold:

(SP1) Every triangle isomorphic to a distinguished one is distinguished. For $X \in \mathcal{U}, 0 \rightarrow X \stackrel{\text { id }}{\rightarrow} X \rightarrow 0$ is a distinguished triangle. Every morphism $u$ : $X \rightarrow Y$ can be completed to a distinguished triangle $X \stackrel{u}{\rightarrow} Y \rightarrow Z \rightarrow T X$.

(SP2) If $X \stackrel{u}{\rightarrow} Y \rightarrow Z \rightarrow T X$ is a distinguished triangle in $\mathcal{U}$, then so is $Y \rightarrow$ $Z \rightarrow T X \stackrel{T u}{\rightarrow} T Y$

$(\mathrm{SP} 3)=(\mathrm{TR} 3)$ in Verdier's loc. cit.

$(\mathrm{SP} 4)=(\mathrm{TR} 4)$ in Verdier's loc. cit.

The main difference with triangulated categories is that the translation functor in a suspended category may not have an inverse, and therefore some objects can not be shifted back. The formulation of axioms (SP1) and (SP2) reflects this fact.

In this paper, we will only consider suspended subcategories of a triangulated category $\mathbf{T}$, and we implicitly assume that they are full and that the translation functor is the same for $\mathbf{T}$. If $\left(\mathbf{T}^{\leq 0}, \mathbf{T}^{\geq 0}\right)$ is a $t$-structure, the aisle $\mathbf{T}^{\leq 0}$ is a suspended subcategory of $\mathbf{T}$ whose distinguished triangles are diagrams in $\mathbf{T} \leq 0$ that are distinguished triangles in $\mathbf{T}$ (Proposition 1.1). Moreover, the aisle $\mathbf{T} \leq 0$ determines the $t$-structure because the co-aisle $\mathbf{T}^{\geq 0}$ is recovered as $\left(\mathbf{T}^{\leq 0}\right)^{\perp}[1]$. The terminology "aisle" and "co-aisle" comes from [KV2].

The following observation that sent us towards the right direction for our objective is the following result, due to Keller and Vossieck:

Theorem 1.2 ([KV2, Section 1]). A suspended subcategory $\mathcal{U}$ of a triangulated category $\mathbf{T}$ is an aisle (i.e., $\left(\mathcal{U}, \mathcal{U}^{\perp}[1]\right)$ is a t-structure on $\left.\mathbf{T}\right)$ if and only if the canonical inclusion functor $\mathcal{U} \hookrightarrow \mathbf{T}$ has a right adjoint.

Our arguments, however, are logically independent of this fact. The knowledgeable reader will recognize a localization situation: to construct a $t$-structure, one basically looks for a right adjoint to the inclusion of a subcategory. In the case of 
triangulated localizations a basic concept is localizing subcategories: those triangulated subcategories stable for coproducts. We will use the analogous concept in the context of $t$-structures.

To respect Keller and Vossieck's terminology we will call pre-aisle a suspended subcategory $\mathcal{U}$ of a triangulated category $\mathbf{T}$ where the triangulation in $\mathcal{U}$ is given by the triangles that are distinguished in $\mathbf{T}$ and the shift functor is induced by the one in $\mathbf{T}$. We see easily that to check that a full subcategory $\mathcal{U}$ of $\mathbf{T}$ is a pre-aisle, it is enough to verify that

- For any $X$ in $\mathcal{U}, X[1]$ is also in $\mathcal{U}$.

- Given a distinguished triangle $X \rightarrow Y \rightarrow Z \rightarrow X[1]$, if $X$ and $Z$ belong to $\mathcal{U}$, then so does $Y$.

Once these two facts hold for $\mathcal{U}$, the verification of axioms (SP1)-(SP4) is immediate.

Let $\mathcal{U}$ be an aisle, i.e., a suspended subcategory of $\mathbf{T}$ such that $\left(\mathcal{U}, \mathcal{U}^{\perp}[1]\right)$ is a $t$-structure. We will denote by $\tau_{\mathcal{U}}^{\leq n}$ and $\tau_{\mathcal{U}}^{\geq n}(n \in \mathbb{Z})$ the truncation functors associated to this $t$-structure, i.e., the corresponding adjoints to the canonical inclusions $\mathcal{U}[-n] \hookrightarrow \mathbf{T}$ and $\mathcal{U}^{\perp}[1-n] \hookrightarrow \mathbf{T}$, respectively.

Lemma 1.3. Let $\mathbf{T}$ be a triangulated category and let $\mathcal{U}$ be an aisle. If a family of objects of $\mathcal{U}$ has a coproduct in $\mathbf{T}$, then it already belongs to $\mathcal{U}$.

Proof. For $Y$ in $\mathbf{T}$,

$$
\operatorname{Hom}\left(\bigoplus X_{i}, \tau_{\mathcal{U}}^{\geq 1} Y\right)=\prod \operatorname{Hom}\left(X_{i}, \tau_{\mathcal{U}}^{\geq 1} Y\right)
$$

so if $\tau_{\mathcal{U}}^{\geq 1} X_{i}=0$ for every $i$, then $\tau_{\mathcal{U}}^{\geq 1}\left(\bigoplus X_{i}\right)=0$. Therefore $\bigoplus X_{i}$ is in $\mathcal{U}$.

The following fact was observed first in the case of localizing subcategories by Bökstedt and Neeman in $\mathrm{BN}$.

Corollary 1.4. A direct summand of an object in an aisle $\mathcal{U}$ belongs to $\mathcal{U}$.

Proof. Use the argument in loc. cit. or, alternatively, Eilenberg's swindle, as in AJS footnote, p. 227].

If $\mathcal{U}$ is a pre-aisle in $\mathbf{T}$ stable for (arbitrary) coproducts in $\mathbf{T}$, then it will be called cocomplete. From the previous discussion, we see that in triangulated categories in which coproducts exist, aisles are cocomplete pre-aisles. Specifically, we try to address the following:

Problem. For a triangulated category $\mathbf{T}$, give conditions that guarantee that a cocomplete pre-aisle is an aisle.

In this paper, we will solve this problem in two cases. Let $\mathcal{A}$ be a Grothendieck category, which means $\mathcal{A}$ is an abelian category with a generator $U$ and exact (setindexed) filtered direct limits. We will give a sufficient condition for a cocomplete pre-aisle to be an aisle for the derived category of $\mathcal{A}$. This means that there is a set (as opposed to a class) of objects that "generates" the pre-aisle. To fix notation, denote by $\mathbf{C}(\mathcal{A})$ the category of complexes of objects of $\mathcal{A}$ (also a Grothendieck category). Denote by $\mathbf{K}(\mathcal{A})$ and $\mathbf{D}(\mathcal{A})$ the homotopy and derived categories of $\mathcal{A}$, with their usual structure of triangulated categories. The proof will rely on a construction by homotopy limits of diagrams made by maps in $\mathbf{C}(\mathcal{A})$ rather than in $\mathbf{D}(\mathcal{A})$; so it cannot be transposed to a general triangulated category. However, 
if the set that "generates" the cocomplete pre-aisle is made of compact objects (see below), then by a similar but simpler argument (possibly well-known), the analogous result is obtained for any triangulated category. We will treat this case in an appendix.

\section{Cocomplete PRE-Aisles AND homotopy COLIMits}

In this section we will show that homotopy colimits of objects in a cocomplete pre-aisle $\mathcal{U}$ of $\mathbf{D}(\mathcal{A})$ belong to $\mathcal{U}$. We will follow the strategy in [AJS for localizing subcategories. So the proofs will be only sketched as briefly as possible.

For convenience to the reader, let us recall the construction of the homotopy colimit of a diagram of complexes as used in [AJS]. Let $G=\left\{G_{s}, \mu_{s t} / s \leq t \in \Gamma\right\}$ be a filtered directed system of $\mathbf{C}(\mathcal{A})$. Let $r>0$ and $s \in \Gamma$. $W_{s}^{r}$ is the set of chains in the ordered set $\Gamma$ of length $r$ that start in $s$. Define a bicomplex $B(G)$ by $B(G)^{k j}:=0$ if $k>0, B(G)^{0 j}:=\bigoplus_{s \in \Gamma} G_{s}^{j}$ and $B(G)^{k j}:=\bigoplus_{s \in \Gamma, w \in W_{s}^{-k}} G_{s, w}^{j}$ if $k<0$, where by $G_{s, w}^{j}$ we denote $G_{s}^{j}$ indexed by a (fixed) chain $w$ of $W_{s}^{-k}$. If $k<0$, we will denote a map from the generator $x: U \rightarrow G_{s, w}^{j} \rightarrow B(G)^{k j}$ by $\left(x ; s<s_{1}<\cdots<s_{-k}\right)$ where $w$ is the chain $s<s_{1}<\cdots<s_{-k}$ and then the horizontal differential $d_{1}$ is defined by the following formula:

$$
\begin{aligned}
& d_{1}^{k j}\left(x ; s<s_{1}<\cdots<s_{-k}\right) \\
& :=\left(\mu_{s s_{1}}(x) ; s_{1}<\cdots<s_{-k}\right)+\sum_{i=1}^{-k}\left((-1)^{i} x ; s<\cdots<\check{s}_{i}<\cdots<s_{-k}\right),
\end{aligned}
$$

where the symbol $\check{s_{i}}$ means that $s_{i}$ is suppressed from the chain. If $k \geq 0$, then $d_{1}$ is 0 . The differential $d_{2}$ is induced by that of the complexes $G_{s}$. The homotopy direct limit of the system is defined as the totalization (by coproducts) of the bicomplex $B(G)$, and we denote it by $\underset{s \in \Gamma}{\operatorname{holim}} G_{s}$.

Lemma 2.1. Let $G=\left\{G_{n} / n \in \mathbb{N}\right\}$ be a directed system in $\mathbf{C}(\mathcal{A})$. Let $\mathcal{U}$ be a cocomplete pre-aisle of $\mathbf{D}(\mathcal{A})$. If $G_{n} \in \mathcal{U}$, for every $n \in \mathbb{N}$, then

$$
\varliminf_{n \in \mathbb{N}} G_{n} \in \mathcal{U}
$$

where the limit is taken in $\mathbf{C}(\mathcal{A})$.

Proof. Consider the Milnor distinguished triangle in $\mathbf{D}(\mathcal{A})$ ([AJS, 3.3]),

$$
\bigoplus_{n \in \mathbb{N}} G_{n} \stackrel{1-\mu}{\longrightarrow} \bigoplus_{n \in \mathbb{N}} G_{n} \longrightarrow \lim _{n \in \mathbb{N}} G_{n} \stackrel{+}{\longrightarrow}
$$

$\mathcal{U}$ being cocomplete, every $G_{n}$ is in $\mathcal{U}$. So the first two objects in this triangle are in $\mathcal{U}$ and therefore the third, because a pre-aisle is stable for mapping cones.

Remark. For a directed system $\left\{G_{n} / n \in \mathbb{N}\right\}$ in a triangulated category $\mathbf{T}$, there is a construction of a homotopy direct limit by Bökstedt and Neeman that makes sense for any triangulated category (see $[\mathrm{BN}]$ ). It is defined by the triangle

$$
\bigoplus_{n \in \mathbb{N}} G_{n} \stackrel{1-\mu}{\longrightarrow} \bigoplus_{n \in \mathbb{N}} G_{n} \longrightarrow C(1-\mu) \stackrel{+}{\longrightarrow},
$$


where $C(1-\mu)$ denotes the mapping cone of $1-\mu$. It is clear that given a cocomplete pre-aisle $\mathcal{U}$ in $\mathbf{T}$, if every $G_{n} \in \mathcal{U}$, then $C(1-\mu) \in \mathcal{U}$. Moreover, by [AJS, Theorem 2.2 , we have the isomorphism in $\mathbf{D}(\mathcal{A})$,

$$
\operatorname{holim}_{n \in \mathbb{N}} G_{n} \stackrel{\sim}{\rightarrow} \lim _{n \in \mathbb{N}} G_{n} .
$$

Also, it is clear that

$$
C(1-\mu) \stackrel{\sim}{\rightarrow} \lim _{n \in \mathbb{N}} G_{n} .
$$

Thus, it will do no harm to identify these three objects if one is working inside $\mathbf{D}(\mathcal{A})$.

Proposition 2.2. Let $\mathcal{U}$ be a cocomplete pre-aisle of $\mathbf{D}(\mathcal{A})$. Let $\Gamma$ be a filtered ordered set and $G=\left\{G_{s}, \mu_{s t} / s \leq t \in \Gamma\right\}$ be a directed system in $\mathbf{C}(\mathcal{A})$ such that $G_{s}$ belongs to $\mathcal{U}$ for every $s \in \Gamma$. Then

$$
\underset{\overrightarrow{s \in \Gamma}}{\operatorname{holim}} G_{s}
$$

also belongs to $\mathcal{U}$.

Proof. By definition,

$$
\underset{\substack{s \in \Gamma}}{\operatorname{holim}} G_{s}=\operatorname{Tot}\left(B(G)^{\cdot *}\right),
$$

where $B^{*}=B(G)^{\cdot}$ is a bicomplex whose columns $B(G)^{i}$ are coproducts of the objects in the system; so they belong to $\mathcal{U}$. By [AJS, Lemma 3.2], there is sequence $F_{n}$ of subcomplexes of $\operatorname{Tot}\left(B(G)^{\cdot *}\right)$ such that

$$
\operatorname{Tot}\left(B(G)^{\cdot \cdot}\right)=\varliminf_{n \in \mathbb{N}} F_{n}
$$

in $\mathbf{C}(\mathcal{A})$. So by the previous lemma we only have to show that every $F_{n}$ is in $\mathcal{U}$ for every $n \in \mathbb{N}$. Indeed, $F_{0}=B(G)^{0}$, a coproduct of objects of $\mathcal{U}$. For $n>0$, take $\nu_{n}$ to be a graded splitting of the inclusion $F_{n-1} \subset B(G)^{-n+1 \cdot}[n-1]$. The composition

$$
B^{-n \cdot}[n-1] \stackrel{d_{1}[n-1]}{\longrightarrow} B^{-n+1} \cdot[n-1] \stackrel{\nu_{n}}{\longrightarrow} F_{n-1}
$$

defines a map of complexes $h_{n}$, and it can be completed to a triangle

$$
B^{-n \cdot}[n-1] \stackrel{h_{n}}{\longrightarrow} F_{n-1}^{\cdot} \longrightarrow F_{n}^{\cdot} \stackrel{+}{\longrightarrow} .
$$

So, $F_{n}$ is the cone of a map from a positive shift of a column of $B(G)^{\cdots}$ to $F_{n-1}$, which belongs to $\mathcal{U}$ by induction; therefore, $F_{n} \in \mathcal{U}$ by Lemma 2.1

\section{Construction of $t$-Structures}

Let $R$ be a ring. In this paper, we will only consider associative rings with unit. Denote by $R$-Mod the category of left $R$-modules. As usual, we abbreviate $\mathbf{C}(R$-Mod $), \mathbf{K}(R$-Mod $)$, and $\mathbf{D}(R$-Mod) by $\mathbf{C}(R), \mathbf{K}(R)$, and $\mathbf{D}(R)$, respectively. We denote by $Q_{R}: \mathbf{K}(R) \rightarrow \mathbf{D}(R)$ the canonical functor.

Lemma 3.1. Let $\mathcal{S}=\left\{E_{\alpha} / \alpha \in A\right\}$ be a set of objects of a triangulated category $\mathbf{T}$ with coproducts. Let $\mathcal{U}$ be the smallest cocomplete pre-aisle that contains the objects in $\mathcal{S}$. The groups $\operatorname{Hom}_{\mathbf{T}}\left(E_{\alpha}[j], B\right)=0$ for every $j \geq 0$ and $\alpha \in A$ if, and only if, $B$ is in $\mathcal{U}^{\perp}$. 
Proof. The "if" part is trivial. Let us prove the "only if" part. Suppose that $B \in \mathbf{T}$ is such that $\operatorname{Hom}_{\mathbf{T}}\left(E_{\alpha}[j], B\right)=0$ for every $j \geq 0$ and $\alpha \in A$. We have to show that $\operatorname{Hom}_{\mathbf{T}}(X, B)=0$ for every $X \in \mathcal{U}$. Let $\mathcal{V}$ be the full subcategory of $\mathcal{U}$ whose objects $X$ are such that $\operatorname{Hom}_{\mathbf{T}}(X[j], B)=0$, for every $j \geq 0$. $\mathcal{V}$ is clearly stable for positive shiftings and for extensions. Finally, $\mathcal{V}$ is stable for coproducts, because

$$
\operatorname{Hom}_{\mathbf{T}}\left(\bigoplus_{i \in I} X_{i}[j], B\right) \simeq \prod_{i \in I} \operatorname{Hom}_{\mathbf{T}}\left(X_{i}[j], B\right) .
$$

Now, $\mathcal{S}$ is contained in $\mathcal{V}$; therefore, $\mathcal{V}=\mathcal{U}$.

Proposition 3.2. Let $\mathcal{U}$ be the smallest cocomplete pre-aisle of $\mathbf{D}(R)$ that contains an object $E \in \mathbf{C}(R)$. Then $\mathcal{U}$ is an aisle in $\mathbf{D}(R)$.

Proof. First, we can assume that the complex $E$ is K-projective by the well-known existence of resolutions (see, for instance, AJS, Proposition 4.3]). Therefore, for $k \in \mathbb{Z}$ every $E[k]$ is also K-projective and maps from $E[k]$ in the derived category are represented by actual maps of complexes. Observe that the conditions (t1) and (t2) of the definition of $t$-structure hold automatically for $\left(\mathcal{U}, \mathcal{U}^{\perp}[1]\right)$; therefore, it is enough to prove (t3), i.e., to construct for any $M \in \mathbf{C}(R)$ a distinguished triangle $N \rightarrow M \rightarrow B \stackrel{+}{\rightarrow}$ with $N \in \mathcal{U}$ and $B \in \mathcal{U}^{\perp}$.

The construction of $B$ is by a transfinite induction and is parallel to the construction given in the proof of [AJS, Proposition, 4.5]. Let $\gamma$ be the least ordinal such that $\#(\gamma)>\# \bigcup_{p \in \mathbb{Z}} E^{p}$ and let $I$ be a set of ordinals that contains $\gamma$.

Let 0 be the minimum of $I$. We define $B_{0}:=M$.

If $s \in I$ has a predecessor $s-1$, suppose by induction that $B_{s-1}$ is already constructed. Take $\Omega_{s-1}:=\bigcup_{k \in \mathbb{N}} \operatorname{Hom}_{\mathbf{C}(R)}\left(E[k], B_{s-1}\right)$, and let $\alpha_{s-1}: \bigoplus_{\Omega_{s-1}} E[k] \rightarrow$ $B_{s-1}$ be given by the universal property of the coproduct. We define $\mu_{s-1 s}$ : $B_{s-1} \rightarrow B_{s}$ by the canonical distinguished triangle:

$$
\bigoplus_{\Omega_{s-1}} E[k] \stackrel{\alpha_{s-1}}{\longrightarrow} B_{s-1} \stackrel{\mu_{s-1 s}}{\longrightarrow} B_{s} \longrightarrow\left(\bigoplus_{\Omega_{s-1}} E[k]\right)[1],
$$

where $B_{s}$ is the mapping cone of $\alpha_{s-1}$. For any $i<s$, let $\mu_{i s}:=\mu_{s-1 s} \circ \mu_{i s-1}$.

If $s \in I$ has no predecessor, take

$$
B_{s}:=\varliminf_{i<s} B_{i} \text { and for } i<s, \mu_{i s}=\varliminf_{i<j<s} \mu_{i j},
$$

where both limits are constructed as objects and maps in $\mathbf{C}(R)$.

We have a directed system in $\mathbf{C}(R),\left\{B_{s}, \mu_{s t} / s \leq t \in I\right\}$, such that every $\mu_{s t}$ is semi-split and if $f \in \operatorname{Hom}_{\mathbf{C}(R)}\left(E[k], B_{s}\right)$, where $s<t$ and $k \in \mathbb{N}$, then $\mu_{s t} \circ f$ is homotopic to zero.

We have to show that $B:=B_{\gamma}$ belongs to $\mathcal{U}^{\perp}$. By the previous lemma $(\mathcal{S}=\{E\})$ it is enough to show that $\operatorname{Hom}_{\mathbf{D}(R)}(E[k], B)=0$, for each $k \in \mathbb{N}$. Let $g: E[k] \rightarrow B$ be a map of complexes. We will show that $g$ is homotopic to zero. Let $B_{i}^{\prime}:=$ $B_{i} \cap \operatorname{Im}(g)$. The ordinal $\gamma$ is a cardinal; so it has no predecessor and

$$
\varliminf_{i<\gamma} B_{i}^{\prime}=\operatorname{Im}(g) \text {. }
$$

We claim that there is an index $s_{0}<\gamma$ such that

$$
\varliminf_{i<s_{0}} B_{i}^{\prime}=B_{s_{0}}^{\prime} .
$$


Thus, there is an $s_{0} \in I$ such that $g$ factors through $\mu_{s_{0} \gamma}$ and, by the properties of our directed system, $g$ is homotopic to zero.

Let us show the claim now. Arguing by contradiction, suppose that for every $s \in I$, there is a $t>s$ such that the map $B_{s}^{\prime} \hookrightarrow B_{t}^{\prime}$ is not an epimorphism. Define sets $J_{s}:=\left\{r \geq s / B_{r}^{\prime} \hookrightarrow B_{r+1}^{\prime}\right.$ is not an epimorphism $\}$. Each $J_{s}$ is nonempty; so $J:=J_{0}$ is a cofinal subset of $I$. But $\gamma$ is a regular ordinal by Hausdorff's theorem ([L] 3.11 Proposition, p. 135]), and, therefore, $\#(J)=\#(\gamma)$.

On the other hand, we can define a map

$$
\phi: J \rightarrow \bigcup_{p \in \mathbb{Z}} \operatorname{Im}(g)^{p}
$$

by $\phi(s)=\alpha_{s}$ where $\alpha_{s} \in \bigcup_{p \in \mathbb{Z}} B^{\prime p}{ }_{s+1}$, but $\alpha_{s} \notin \bigcup_{p \in \mathbb{Z}} B^{\prime p}{ }_{s}$. The map $\phi$ is injective and $\operatorname{Im}(g)$ is a quotient of $E[k]$; therefore, $\#(J) \leq \#\left(\bigcup_{p \in \mathbb{Z}} \operatorname{Im}(g)^{p}\right)<\#(\gamma)$, a contradiction.

The only thing left to prove is that in the triangle $N \rightarrow M \rightarrow B \stackrel{+}{\rightarrow}$ we have that $N \in \mathcal{U}$. For each $i<\gamma$, it is clear that defining $N_{i}$ by the distinguished triangle

$$
N_{i} \longrightarrow M \stackrel{\mu_{0 i}}{\longrightarrow} B_{i} \longrightarrow N_{i}[1]
$$

we can take $N_{i}[1]=\operatorname{Coker}\left(\mu_{0 i}\right)$ (in $\mathbf{C}(R)$ ) and that we have $N=\varliminf_{i<\gamma} N_{i}$, where for $s \leq t$ the transition maps $N_{s} \rightarrow N_{t}$ of this system are induced by $\mu_{s t}: B_{s} \rightarrow B_{t}$. We will check that $N_{i} \in \mathcal{U}$ for every $i \in I$ and this will finish the proof.

First, $N_{0}=0$; so it belongs to $\mathcal{U}$. If $i$ has a predecessor, consider the triangles like (3.1) whose second maps are $\mu_{0 i-1}$ and $\mu_{0 i}$. Consider also the distinguished triangle

$$
\bigoplus_{\Omega_{i-1}} E[k] \longrightarrow B_{i-1} \stackrel{\mu_{i-1} i}{\longrightarrow} B_{i} \longrightarrow\left(\bigoplus_{\Omega_{i-1}} E[k]\right)[1] .
$$

We have $\mu_{i-1 i} \circ \mu_{0 i-1}=\mu_{0 i}$. We can apply the octahedral axiom which gives us a distinguished triangle

$$
N_{i-1} \longrightarrow N_{i} \longrightarrow \bigoplus_{\Omega_{i-1}} E[k] \longrightarrow N_{i-1}[1]
$$

where $N_{i-1}$ and $\bigoplus_{\Omega_{i-1}} E[k]$ belong to $\mathcal{U}$. This implies $N_{i}$ is also in $\mathcal{U}$. Finally, if $i$ has no predecessor, then $N_{i}$ is a direct limit of complexes $N_{s}$ that belong to $\mathcal{U}$ by induction.

Applying [AJL, Theorem 2.2] we have that

$$
\underset{s<i}{\operatorname{holim}} N_{s} \simeq \underset{s<i}{\lim _{s}} N_{s}
$$

and by Proposition 2.2 , the homotopy limit belongs to $\mathcal{U}$. So we reach the desired conclusion.

Given an aisle $\mathcal{U}$ defined as the smallest that contains an object $E$, we will denote the truncation functors associated to the $t$-structure $\left(\mathcal{U}, \mathcal{U}^{\perp}[1]\right)$ by $\tau_{\bar{E}}^{\leq n}=\tau_{\mathcal{U}}^{\leq n}$ and $\tau_{\bar{E}}^{\geq n}=\tau_{\overline{\mathcal{U}}}^{\geq n}(n \in \mathbb{Z})$.

Remark. The hypothesis of the previous result is extended immediately to several generators, the smallest cocomplete pre-aisle that contains a set of objects agrees with the one that contains its coproduct because being cocomplete implies being stable for direct summands (Corollary 1.4). 
3.3. Our next step is to generalize the previous result to the derived category of a Grothendieck category $\mathcal{A}$. By the Gabriel-Popesco embedding ([GP $]$; see also $[\mathrm{St}$. Ch. X, Theorem 4.1]), $\mathcal{A}$ is a quotient (in the sense of abelian categories) of $R$-Mod by a thick subcategory whose objects are the torsion objects of a hereditary torsion theory, where $R$ is $\operatorname{End}_{\mathcal{A}}(U)$ and $U$ denotes a generator of $\mathcal{A}$. This means there are a couple of functors

$$
R \text {-Mod } \underset{i}{\stackrel{a}{\rightleftarrows}} \mathcal{A}
$$

where $a$ is an exact functor and $i$ is left-exact full, faithful and right adjoint to $a$. The torsion objects are those $R$-modules that are sent to zero by $a$.

By the existence of Bousfield localization in the derived category of a ring ( Proposition 4.5]), the situation extends to derived categories. Precisely, there is a diagram of triangulated functors:

$$
\mathbf{D}(R) \underset{\mathbf{i}}{\stackrel{\mathbf{a}}{\rightleftarrows}} \mathbf{D}(\mathcal{A})
$$

The functor $a$ is exact; therefore, it has a canonical extension to the derived category, which we denote $\mathbf{a}$. This functor has a right adjoint $\mathbf{i}$ which extends $i$ whose existence is not immediate but follows from [AJS, Proposition 5.1]. This says that there is a localizing subcategory $\mathcal{L}_{\mathcal{A}}$ of $\mathbf{D}(R)$ generated by a set of complexes such that the quotient $\mathbf{D}(R) / \mathcal{L}_{\mathcal{A}}$ is equivalent to $\mathbf{D}(\mathcal{A})$ and the associated localization functor is identified with $\mathbf{i} \circ \mathbf{a}$. Note that $\mathbf{a} \circ \mathbf{i}=\operatorname{id}_{\mathbf{D}(\mathcal{A})}$. Let $F$ be a generator of the localizing subcategory $\mathcal{L}_{\mathcal{A}}$. Using Lemma 3.1 we can check that the smallest cocomplete pre-aisle that contains the set of objects $\{F[-n] / n \in \mathbb{N}\}$ is in fact $\mathcal{L}_{\mathcal{A}}$. This is an essential point for the proof of the next result.

Theorem 3.4. Let $\mathcal{U}$ be the smallest cocomplete pre-aisle of $\mathbf{D}(\mathcal{A})$ that contains an object $E \in \mathbf{C}(\mathcal{A})$. Then $\mathcal{U}$ is an aisle in $\mathbf{D}(\mathcal{A})$.

Proof. Keeping the notation of the previous discussion let $\mathcal{U}^{\prime}$ be the smallest cocomplete pre-aisle in $\mathbf{D}(R)$ that contains $\mathbf{i} E$ and $F[-n]$ for all $n \in \mathbb{N}$. Then by Proposition 3.2 it is an aisle. Consequently, for an object $M \in \mathbf{D}(\mathcal{A})$, there is a distinguished triangle in $\mathbf{D}(R)$,

$$
N_{0} \longrightarrow \mathbf{i}(M) \longrightarrow B_{0} \stackrel{+}{\longrightarrow},
$$

where $N_{0} \in \mathcal{U}^{\prime}$ and $B_{0} \in \mathcal{U}^{\prime \perp} \subset \mathcal{L}_{\mathcal{A}}^{\perp}$. This gives a triangle in $\mathbf{D}(\mathcal{A})$ :

$$
\mathbf{a}\left(N_{0}\right) \longrightarrow M \longrightarrow \mathbf{a}\left(B_{0}\right) \stackrel{+}{\longrightarrow} \text {. }
$$

The pre-aisle $\mathcal{U}$ is the essential image by a of $\mathcal{U}^{\prime}$; so it is clear that $\mathbf{a}\left(N_{0}\right)$ belongs to $\mathcal{U}$. We check that $\mathbf{a}\left(B_{0}\right)$ is in $\mathcal{U}^{\perp}$. Let $j \geq 0$. Using the fact that $E$ comes from $\mathbf{D}(\mathcal{A})$ and $B_{0}$ is $\mathcal{L}_{\mathcal{A}}$-local, we see that

$$
\left.\operatorname{Hom}_{\mathbf{D}(\mathcal{A})}\left(E[j], \mathbf{a} B_{0}\right) \simeq \operatorname{Hom}_{\mathbf{D}(R)}\left(\mathbf{i} E[j], \mathbf{i a} B_{0}\right)\right)=0 .
$$

Applying Lemma 3.1 again, we conclude.

\section{Boundedness And $t$-STRuCtures}

From here on, we will apply our method of construction of $t$-structures to the problem of characterizing equivalences of bounded derived categories. In this section and the next we will deal with the issue of when a $t$-structure defined on the full category $\mathbf{D}(\mathcal{A})$ restricts to a subcategory defined through a boundedness condition. 
Proposition 4.1. Let $E \in \mathbf{D}^{-}(\mathcal{A})$. The t-structure defined by $E$ restricts to $\mathbf{D}^{-}(\mathcal{A})$; in other words, the associated truncation functors, $\tau_{\bar{E}}^{\geq n}$ and $\tau_{\bar{E}}^{\leq n}(n \in \mathbb{Z})$ take upper bounded objects to upper bounded objects.

Proof. It is enough to check the assertion for $\tau_{\bar{E}}^{\leq 0}$ and $\tau_{\bar{E}}^{\geq 1}$. Note that the aisle $\mathcal{U}$ generated by $E$ is such that $\mathcal{U} \subset \mathbf{D}^{-}(\mathcal{A})$, because the operations that allow us to construct $\mathcal{U}$ from $E$ (translation and extension) preserve this condition; therefore, for every $M \in \mathbf{D}^{-}(\mathcal{A}), \tau_{\bar{E}}^{\leq 0}(M) \in \mathcal{U} \subset \mathbf{D}^{-}(\mathcal{A})$. Now considering the triangle

$$
\tau_{E}^{\leq 0} M \longrightarrow M \longrightarrow \tau_{E}^{\geq 1} M \stackrel{+}{\longrightarrow}
$$

$M$ and $\tau_{\bar{E}}^{\leq 0} M$ are in $\mathbf{D}^{-}(\mathcal{A})$; therefore, $\tau_{\bar{E}}^{\geq 1} M \in \mathbf{D}^{-}(\mathcal{A})$.

Our next task will be to get conditions for the restriction of a $t$-structure to $\mathbf{D}^{+}(\mathcal{A})$. In the rest of this section we will treat the case of derived categories of modules.

4.2. We need a standard definition in the theory of triangulated categories. Let $\mathbf{T}$ be a triangulated category. An object $E$ of $\mathbf{T}$ is called compact if the functor $\operatorname{Hom}_{\mathbf{T}}(E,-)$ commutes with arbitrary coproducts. Another way of expressing the condition is that a map from $E$ to a coproduct factors through a finite subcoproduct.

The following is Rickard's criterion for compact objects in derived categories over rings. See [R, Proposition 6.3 and its proof]. Using results of Neeman, we are able to give a simpler proof.

Let $\mathbf{T}$ be a triangulated category. A triangulated subcategory $\mathcal{S} \subset \mathbf{T}$ is called thick if a direct summand of an object of $\mathcal{S}$ also belongs to $\mathcal{S}$. Observe that a localizing subcategory is thick, because being closed for coproducts implies being closed for direct summands, as follows from Corollary 1.4.

Lemma 4.3. Let $R$ be a ring. In $\mathbf{D}(R)$ the compact objects are those isomorphic to a bounded complex of finite-type projective modules.

Proof. The triangulated category $\mathbf{D}(R)$ is generated by $R$ that is trivially compact and bounded. We apply Neeman's result ([N] Lemma 2.2]) which states that the smallest thick subcategory of a compactly generated triangulated category that contains all the translations of a compact generator is the full subcategory whose objects are the compact ones. Therefore, the full subcategory of compact objects is the smallest thick subcategory of $\mathbf{D}(R)$ that contains $R$. Now the full subcategory of bounded complexes of projectives is thick, because it is stable for triangles and direct summands, and all of its objects are compact as can be easily checked; therefore, it agrees with the subcategory of compact objects.

4.4. In a triangulated category $\mathbf{T}$, a family of objects $\left\{E_{\alpha}\right\}_{\alpha \in A}$ is called a generating family if for every $M \in \mathbf{T}, M \neq 0$ there is a $j \in \mathbb{Z}$ and an $\alpha \in A$ such that $\operatorname{Hom}_{\mathbf{T}}\left(E_{\alpha}[j], M\right) \neq 0$. If the family is formed by a single object, then this object is usually called a generator.

The following result will be key for applications.

Proposition 4.5. Let $R$ be a ring. Let $E$ be a compact generator of $\mathbf{D}(R)$, and denote by $\mathcal{U}$ the aisle generated by $E$. Then every object $M \in \mathcal{U}^{\perp}$ belongs to $\mathbf{D}^{+}(R)$. 
Proof. Fix $M \in \mathcal{U}^{\perp}$. Let $\mathcal{S}_{M}$ be the full subcategory of $\mathbf{D}(R)$ whose objects are those $N$ in $\mathbf{D}(R)$ such that $\mathbf{R H o m}_{R}(N, M) \in \mathbf{D}^{+}(\mathbb{Z})$.

The subcategory $\mathcal{S}_{M}$ is a thick subcategory of $\mathbf{D}(R)$. Indeed, it is clearly triangulated, and a direct summand $N^{\prime}$ of an object $N$ of $\mathcal{S}_{M}$ also belongs to $\mathcal{S}_{M}$ because $\mathbf{R H o m}_{R}\left(N^{\prime}, M\right)$ is a direct summand of $\mathbf{R H o m}_{R}(N, M)$. The object $E$ and all of its translations $E[k](k \in \mathbb{Z})$ belong to $\mathcal{S}_{M}$ because $M \in \mathcal{U}^{\perp}$, which implies that for all $j \leq 0$,

$$
H^{j}\left(\mathbf{R H o m}_{R}(E, M)\right)=\operatorname{Hom}_{\mathbf{D}(R)}(E[-j], M)=0 ;
$$

so $\mathbf{R H o m}_{R}(E, M) \in \mathbf{D}^{\geq 1}(\mathbb{Z})$.

We have shown that $\mathcal{S}_{M}$ is a thick subcategory of $\mathbf{D}(R)$ that contains all translations of a compact generator. Again, we apply Neeman's result ([N, Lemma 2.2.]). It follows that all the compact objects are contained in $\mathcal{S}_{M}$, in particular, $R \in \mathcal{S}_{M}$. But this means that $\mathbf{R H o m}_{R}(R, M) \in \mathbf{D}^{+}(\mathbb{Z})$; in other words, $H^{j}(M)=H^{j}\left(\mathbf{R H o m}_{R}(R, M)\right)=0$, for $j \ll 0$, i.e., $M \in \mathbf{D}^{+}(R)$, as desired.

Theorem 4.6. Let $R$ be a ring and $E$ a compact generator of $\mathbf{D}(R)$. The t-structure defined in $\mathbf{D}(R)$ by $\mathcal{U}$, the smallest aisle that contains $E$, restricts to a $t$-structure on $\mathbf{D}^{\mathrm{b}}(R)$.

Proof. Consider again the triangle associated to $M \in \mathbf{D}(R)$ :

$$
\tau_{\bar{E}}^{\leq 0} M \longrightarrow M \longrightarrow \tau_{\bar{E}}^{\geq 1} M \stackrel{+}{\longrightarrow} .
$$

By Lemma 4.3 we can assume that $E$ is bounded, in particular, $E \in \mathbf{D}^{-}(R)$ and, by Proposition 4.1, the $t$-structure defined by $E$ restricts to $\mathbf{D}^{-}(R)$. This means that when $M \in \mathbf{D}^{\mathrm{b}}(R)$, then both $\tau_{\bar{E}}^{\leq 0} M$ and $\tau_{\bar{E}}^{\geq 1} M \in \mathbf{D}^{-}(R)$.

On the other hand, by Proposition 4.5 we always have that $\tau_{\bar{E}}^{>1} M \in \mathbf{D}^{+}(R)$; therefore, if $M \in \mathbf{D}^{\mathrm{b}}(R)$, then $\tau_{\bar{E}}^{\leq 0} M \in \mathbf{D}^{+}(R)$ also. Putting all this together, $\tau_{\bar{E}}^{\leq 0} M$ and $\tau_{\bar{E}}^{\geq 1} M \in \mathbf{D}^{\mathrm{b}}(R)$.

\section{BOUNDEDNESS FOR QUASI-COHERENT SHEAVES}

The previous result can be extended for sheaves on separated divisorial schemes. Recall that a scheme $\left(X, \mathcal{O}_{X}\right)$ is called divisorial if it is quasi-compact and quasiseparated and has an ample family of line bundles; see [I]. An illuminating example is given by a quasi-projective variety (over a field, say).

Denote by $\mathrm{Qco}(X)$ the category of quasi-coherent sheaves on a scheme $X$. If $X$ is separated, the category $\mathbf{D}(\mathrm{Q} \operatorname{co}(X))$ has an internal tensor functor, $\otimes_{\mathcal{O}_{X}}^{\mathbf{L}}$, that can be computed by quasi-coherent flat resolutions in either variable; see [AJL, 1.1].

Let $d \in \mathbb{N}$. An aisle $\mathcal{U}$ in $\mathbf{D}(\mathrm{Q} \operatorname{co}(X))$ is called $d$-rigid if for every $\mathcal{G} \in \mathcal{U}$ and $\mathcal{F} \in \mathbf{D}^{\leq 0}(\mathrm{Q} \operatorname{co}(X))$ we have that $\mathcal{F} \otimes_{\mathcal{O}_{X}}^{\mathbf{L}} \mathcal{G} \in \mathcal{U}[-d]$. If $\mathcal{U}$ is $d$-rigid for some $d$, we will say simply that $\mathcal{U}$ is rigid. The next result gives a useful characterization of rigid aisles in $\mathbf{D}(\mathrm{Qco}(X))$ when $X$ is a divisorial scheme.

Proposition 5.1. Let $\left(X, \mathcal{O}_{X}\right)$ be a divisorial separated scheme. Let $\mathcal{U}$ be an aisle of $\mathbf{D}(\mathrm{Q} \operatorname{co}(X))$ and $d \in \mathbb{N}$. The following are equivalent:

(i) $\mathcal{U}$ is d-rigid.

(ii) For every $\mathcal{G} \in \mathcal{U}$ and $\mathcal{F} \in \mathrm{Q} \operatorname{co}(X)$ we have that $\mathcal{F} \otimes_{\mathcal{O}_{X}} \mathcal{G} \in \mathcal{U}[-d]$.

(iii) For every $\mathcal{G} \in \mathcal{U}$ and $\mathcal{F}$ a locally free sheaf in $\mathrm{Q} \operatorname{co}(X)$ we have that $\mathcal{F} \otimes_{\mathcal{O}_{X}}$ $\mathcal{G}=\mathcal{F} \otimes_{\mathcal{O}_{X}}^{\mathrm{L}} \mathcal{G} \in \mathcal{U}[-d]$. 
(iv) For every $\mathcal{G} \in \mathcal{U}$, and for every invertible sheaf $\mathcal{L}$ from the ample family of sheaves in $\mathrm{Qco}(X)$, there is a $k_{0} \in \mathbb{N}$ such that $\mathcal{L}^{\otimes k} \otimes_{\mathcal{O}_{X}} \mathcal{G} \in \mathcal{U}[-d]$ if $k<k_{0}$.

Proof. The assertions $(i) \Rightarrow(i i) \Rightarrow(i i i) \Rightarrow(i v)$ are trivial. To prove $(i v) \Rightarrow(i)$ take a complex $\mathcal{F} \in \mathbf{D}^{\leq 0}(\mathrm{Q} \operatorname{co}(X))$. The complex $\mathcal{F} \otimes_{\mathcal{O}_{X}} \mathcal{G}$ can be computed by means of a flat resolution $\mathcal{E} \rightarrow \mathcal{F}$, where each component $\mathcal{E}^{i}$ is an arbitrary coproduct of sheaves $\mathcal{L}^{\otimes k}$ and where $\mathcal{L}$ is an invertible sheaf from the ample family and $k \ll 0$. Therefore, we have the quasi-isomorphism $\mathcal{E} \otimes_{\mathcal{O}_{X}} \mathcal{G} \rightarrow \mathcal{F} \otimes_{\mathcal{O}_{X}}^{\mathbf{L}} \mathcal{G}$. The complex $\mathcal{E} \otimes_{\mathcal{O}_{X}} \mathcal{G}$ is defined as the totalization of the bicomplex $\mathcal{E} \cdot \otimes_{\mathcal{O}_{X}} \mathcal{G}$. Fixing $i \leq 0$, the complex $\mathcal{E}^{i} \otimes_{\mathcal{O}_{X}} \mathcal{G}$ belongs to $\mathcal{U}[-d]$ by $(i v)$ and the previous observation using that an aisle is stable for coproducts and the derived tensor product of sheaves commutes with coproducts. This allows us to apply the same argument as in Proposition 2.2 We define a filtration $\mathcal{H}_{n}$ of $\mathcal{E} \otimes_{\mathcal{O}_{X}} \mathcal{G}$ such that $\mathcal{H}_{0}=\mathcal{E}^{0} \otimes_{\mathcal{O}_{X}} \mathcal{G}$. For $n>0$ there is a map $\nu_{n}$ from which we define $\mathcal{H}_{n}$ inductively by the triangle

$$
\left(\mathcal{E}^{-n} \otimes_{\mathcal{O}_{X}} \mathcal{G}^{\cdot}\right)[n-1] \stackrel{\nu_{n}}{\longrightarrow} \mathcal{H}_{n-1} \longrightarrow \mathcal{H}_{n} \stackrel{+}{\longrightarrow} .
$$

Thus, every $\mathcal{H}_{n} \in \mathcal{U}[-d]$ by induction. Clearly, in $\mathbf{C}(\mathrm{Qco}(X))$,

$$
\underset{n}{\stackrel{\lim }{\longrightarrow}} \mathcal{H}_{n}=\mathcal{E} \otimes_{\mathcal{O}_{X}} \mathcal{G}
$$

and it follows that $\mathcal{E} \otimes_{\mathcal{O}_{X}} \mathcal{G} \in \mathcal{U}[-d]$ by Lemma 2.1

Corollary 5.2. If $X$ is affine, then every aisle in $\mathbf{D}(\mathrm{Qco}(X))$ is rigid.

Proof. In this case $\left\{\mathcal{O}_{X}\right\}$ is an ample family.

5.3. Let $\left(X, \mathcal{O}_{X}\right)$ be a scheme. Let $\mathcal{L}$ be an invertible sheaf on $X$ and $s: \mathcal{O}_{X} \rightarrow \mathcal{L}$ a global section. For any $n \in \mathbb{N}$, there is a map $\mathcal{L}^{\otimes n} \rightarrow \mathcal{L}^{\otimes n+1}$ defined by $t \mapsto t \otimes s$. These maps form a directed system of $\mathcal{O}_{X}$-modules whose limit we will denote

$$
\mathcal{O}_{X}\left[s^{-1}\right]:=\underset{n}{\underset{n}{l}} \mathcal{L}^{\otimes n} .
$$

The notation is explained by the fact that if $X$ is affine, say $X=\operatorname{Spec}(R)$, and $s \in R$ is identified with a global section of the sheaf $\mathcal{O}_{X}=\widetilde{R}$, then $\mathcal{O}_{X}\left[s^{-1}\right]=\widetilde{R_{s}}$.

Lemma 5.4. Let $\left(X, \mathcal{O}_{X}\right)$ be a divisorial separated scheme and $\mathcal{L}$ an invertible sheaf from the given ample family. Let $X_{s}$ be the open set whose complementary set is the set of zeros of a global section $s: \mathcal{O}_{X} \rightarrow \mathcal{L}$. Denote by $j: X_{s} \hookrightarrow X$ the canonical inclusion map. Let $\mathcal{F} \in \mathbf{D}(\mathrm{Q} c o(X))$. Then we have an isomorphism (in $\mathbf{D}(\mathrm{Q} \operatorname{co}(X)))$ :

$$
\mathbf{R} j_{*} j^{*} \mathcal{F} \stackrel{\sim}{\longrightarrow} \mathcal{O}_{X}\left[s^{-1}\right] \otimes \mathcal{O}_{X} \mathcal{F}
$$

Proof. By [I] Lemme 2.2.3.1] the morphism $j: X_{s} \hookrightarrow X$ is affine; therefore, $j_{*}$ : $\mathrm{Qco}\left(X_{s}\right) \rightarrow \mathrm{Qco}(X)$ is exact. Being an open embedding, $j$ is flat; so $j^{*}$ is exact also. Therefore, we have the isomorphism $\mathbf{R} j_{*} j^{*} \mathcal{F} \sim j_{*} j^{*} \mathcal{F}$ for every $\mathcal{F} \in \mathbf{D}(\mathrm{Qco}(X))$. So, it is enough to treat the case in which $\mathcal{F}$ is a single quasi-coherent sheaf. In fact, this is a restatement of [EGA I, 6.8.1].

Consider the canonical commutative diagram

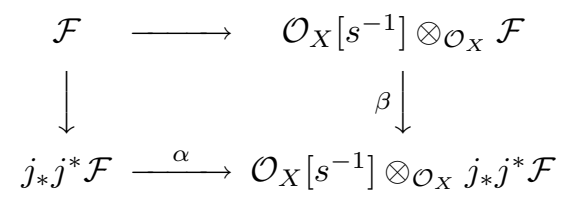


The maps $\alpha$ and $\beta$ are isomorphisms. Indeed, consider a covering of $X$ made up by affine trivialization open subsets, i.e., every $U=\operatorname{Spec}(R)$ in the covering is such that $\left.\mathcal{L}\right|_{U} \sim \widetilde{\rightarrow}$, and the section $\left.s\right|_{U}$ is identified with a certain $f \in R$. Suppose also that $\left.\mathcal{F}\right|_{U} \sim \widetilde{M}$ for a certain $R$-module $M$. Observe that the open set $X_{s} \cap U$ is identified with $\operatorname{Spec} R_{f}$. Thus, $\left.\alpha\right|_{U}$ corresponds to the canonical isomorphism $\widetilde{M_{f}} \tilde{\rightarrow} \widetilde{R_{f}} \otimes_{\left.\mathcal{O}_{X}\right|_{U}} \widetilde{M_{f}}$ and $\left.\beta\right|_{U}$ corresponds to $\widetilde{R_{f}} \otimes_{\left.\mathcal{O}_{X}\right|_{U}} \widetilde{M} \tilde{\rightarrow} \widetilde{R_{f}} \otimes_{\left.\mathcal{O}_{X}\right|_{U}} \widetilde{M_{f}}$

For divisorial schemes there is a characterization of compact objects in $\mathbf{D}_{\mathrm{qc}}(X)$, the category of complexes of sheaves of $\mathcal{O}_{X}$-modules with quasi-coherent homology, due to Thomason and Trobaugh; see [TT, Theorem 2.4.3]. On the other hand, it is well known that for a quasi-compact separated scheme we have an equivalence $\mathbf{D}_{\mathrm{qc}}(X) \simeq \mathbf{D}(\mathrm{Q} \operatorname{co}(X))$; see, for instance, [BN, Corollary 5.5] or, for a different proof, AJL, Proposition 1.3]. We give here a proof of this criterion for $\mathbf{D}(\mathrm{Q} \operatorname{co}(X))$.

Lemma 5.5. Let $\left(X, \mathcal{O}_{X}\right)$ be a divisorial, separated scheme. In $\mathbf{D}(\mathrm{Q} \operatorname{co}(X))$ the compact objects are those isomorphic to bounded complexes of locally free, finite type sheaves.

Proof. Again we apply Neeman's result ([N, Lemma 2.2]) for $\mathbf{D}(\mathrm{Qco}(X))$ and an ample family of invertible sheaves as compact generators. Then we mimic the procedure of Lemma 4.3

Proposition 5.6. Let $\left(X, \mathcal{O}_{X}\right)$ be a divisorial, separated scheme. Let $\mathcal{E}$ be a compact generator of $\mathbf{D}(\mathrm{Q} c o(X))$, and denote by $\mathcal{U}$ the aisle generated by $\mathcal{E}$. Suppose that $\mathcal{U}$ is rigid. Then every object $\mathcal{F} \in \mathcal{U}^{\perp}$ belongs to $\mathbf{D}^{+}(\mathrm{Q} \operatorname{co}(X))$.

Proof. As in the case of Proposition 4.5 we only need to check that

$$
\mathcal{H}^{q}(\mathcal{F})=\mathcal{E}_{x t_{\mathcal{O}_{X}}^{q}}^{q}\left(\mathcal{O}_{X}, \mathcal{F}\right)
$$

vanishes for $q \ll 0$. But this is a local question; therefore, we can cover $X$ by a finite family of open affines, $U_{\lambda}=\operatorname{Spec}\left(R_{\lambda}\right)$, where $\lambda \in\{1, \ldots, n\}$. Denote by $j_{\lambda}$ : $U_{\lambda} \hookrightarrow X$ the canonical inclusion. We choose these open affines as complementary sets of zeros of sections $s_{\lambda}: \mathcal{O}_{X} \rightarrow \mathcal{L}_{\lambda}$ of invertible sheaves $\mathcal{L}_{\lambda}$ from the ample family; $U_{\lambda}$ will be the locus where $s_{\lambda}$ does not vanish. The complex $\mathcal{E}$ restricted to each $\operatorname{Spec}\left(R_{\lambda}\right)$ can be represented by a bounded complex of free $R_{\lambda}$-modules (after refining the covering, if necessary). Define complexes $E_{\lambda}$ and $F_{\lambda}$ of $R_{\lambda}$-modules by $\widetilde{E_{\lambda}}=\left.\mathcal{E}\right|_{\operatorname{Spec}\left(R_{\lambda}\right)}$ and $\widetilde{F_{\lambda}}=\left.\mathcal{F}\right|_{\operatorname{Spec}\left(R_{\lambda}\right)}$.

The complex $E_{\lambda}$ is a compact generator in $\mathbf{D}\left(R_{\lambda}\right)$. Indeed, by Lemma 5.5 the covering by open affines can be chosen in such a way that $E_{\lambda}$ are bounded complexes of projective modules of finite type which are compact as objects of $\mathbf{D}\left(R_{\lambda}\right)$. Let us check that $E_{\lambda}$ is a generator of $\mathbf{D}\left(R_{\lambda}\right)$. For a nonzero object $M$ of $\mathbf{D}\left(R_{\lambda}\right)$, its derived extension $\mathbf{R} j_{\lambda *} \widetilde{M}$ is again nonzero because $j_{\lambda}^{*} \mathbf{R} j_{\lambda *} \widetilde{M}=\widetilde{M} \neq 0$. Therefore, there is a nonzero map $\mathcal{E}[r] \rightarrow \mathbf{R} j_{\lambda *} \widetilde{M}$ for some $r \in \mathbb{Z}$ which, by adjunction, gives a nonzero map $E_{\lambda}[r] \rightarrow M$.

Suppose that $\mathcal{U}$ is $d$-rigid. Let $\mathcal{U}_{\lambda}$ be the aisle of $\mathbf{D}\left(R_{\lambda}\right)$ generated by $E_{\lambda}[d]$. We will see that $F_{\lambda} \in \mathcal{U}_{\lambda}^{\perp}$. We have to check that

$$
\operatorname{Hom}_{\mathbf{D}\left(R_{\lambda}\right)}\left(E_{\lambda}[r], F_{\lambda}\right)=0
$$

for every $r \geq d$. First, again using [AJS, Theorem 2.2], we have that

$$
\underset{n}{\operatorname{holim}} \mathcal{L}^{\otimes n} \stackrel{\sim}{\rightarrow} \underset{n}{\lim } \mathcal{L}^{\otimes n}=\mathcal{O}_{X}\left[s^{-1}\right] .
$$


Now we have the following chain of isomorphisms:

$$
\begin{aligned}
& \operatorname{Hom}_{\mathbf{D}\left(R_{\lambda}\right)}\left(E_{\lambda}[r], F_{\lambda}\right) \stackrel{\sim}{\rightarrow} \operatorname{Hom}_{\mathbf{D}\left(\mathrm{Qco}\left(U_{\lambda}\right)\right)}\left(j_{\lambda}^{*} \mathcal{E}[r], j_{\lambda}^{*} \mathcal{F}\right) \\
& \stackrel{\sim}{\rightarrow} \operatorname{Hom}_{\mathbf{D}(Q \operatorname{Qco}(X))}\left(\mathcal{E}[r], \mathbf{R} j_{\lambda *} j_{\lambda}^{*} \mathcal{F}\right) \\
& \stackrel{\sim}{\rightarrow} \operatorname{Hom}_{\mathbf{D}(\mathrm{Qco}(X))}\left(\mathcal{E}[r], \mathcal{O}_{X}\left[s_{\lambda}^{-1}\right] \otimes \mathcal{F}\right) \\
& \stackrel{\sim}{\rightarrow} \operatorname{Hom}_{\mathbf{D}(\mathrm{Qco}(X))}\left(\mathcal{E}[r],\left(\underset{n}{\operatorname{holim}} \mathcal{L}^{\otimes n}\right) \otimes \mathcal{F}\right) \\
& \stackrel{\sim}{\rightarrow} \underset{n}{\lim _{\longrightarrow}} \operatorname{Hom}_{\mathbf{D}(\mathrm{Qco}(X))}\left(\mathcal{E}[r], \mathcal{L}^{\otimes n} \otimes \mathcal{F}\right) \\
& \stackrel{\sim}{\rightarrow} \underset{n}{\lim _{\mathbf{D}}} \operatorname{Hom}_{\mathrm{Q}(\mathrm{Qco}(X))}\left(\mathcal{E}[r] \otimes \mathcal{L}^{\otimes-n}, \mathcal{F}\right) \\
& =0 \text {, }
\end{aligned}
$$

where the first isomorphism holds because $U_{\lambda}$ is affine, the third using Lemma 5.4 the fourth following the previous remark, the fifth using that $\mathcal{E}$ is a compact object in $\mathbf{D}(\mathrm{Qco}(X))$, and the rest are the usual adjunction maps. The last equality follows from the fact that $\mathcal{U}$ is $d$-rigid.

Now, given $\lambda \in\{1, \ldots, n\}$, Proposition 4.5 gives us a bound on $q$ for the vanishing of $\operatorname{Ext}_{R}^{q}\left(R_{\lambda}, F_{\lambda}\right)$. Since these bounds are in finite quantity, we take the minimum and conclude.

Theorem 5.7. Let $X$ be a separated divisorial scheme $X$. If $\mathcal{E}$ is as in the previous proposition, the $t$-structure defined by $\mathcal{E}$ in $\mathbf{D}(\mathrm{Q} \operatorname{co}(X))$ restricts to a $t$-structure on $\mathbf{D}^{\mathrm{b}}(\mathrm{Q} \operatorname{co}(X))$.

Proof. Mimic the proof of Theorem 4.6 using Proposition5.6 instead of Proposition 4.5 and the fact that $\mathcal{E} \in \mathbf{D}^{-}(\mathrm{Qco}(X))$ by Lemma 5.5 .

\section{Derived Equivalences And $t$-STructures}

First, we need the following easy Lemma.

Lemma 6.1. Let $\mathbf{T}$ be a triangulated category and $\mathcal{U}$ the smallest cocomplete preaisle in $\mathbf{T}$ that contains an object $E$. If $E$ is compact, then $\operatorname{Hom}_{\mathbf{T}}(E, X[j])=0$ for all $j>0$ and all $X \in \mathcal{U}$ if, and only if, $\operatorname{Hom}_{\mathbf{T}}(E, E[j])=0$ for all $j>0$.

Proof. We will use again an idea from a previous lemma. Consider the full subcategory $\mathcal{S}$ whose objects are those $X \in \mathcal{U}$ such that $\operatorname{Hom}_{\mathbf{T}}(E, X[j])=0$ for every $j>0$. Clearly, $\mathcal{S} \subset \mathcal{U}$ is suspended and stable for coproducts ( $E$ is compact); therefore, if $E \in \mathcal{S}$, necessarily $\mathcal{S}=\mathcal{U}$.

6.2. In a triangulated category $\mathbf{T}$, an object $X$ is called exceptional if, and only if,

$$
\operatorname{Hom}_{\mathbf{T}}(X, X[j])=0 \text {, for all } j \neq 0 \text {. }
$$

For instance, an object $M$ in $\mathbf{D}(R)\left(R\right.$ a ring) is exceptional if $\operatorname{Ext}_{R}^{j}(M, M)=0$ for all $j \neq 0$. In the literature, an exceptional compact generator is often called a tilting object.

Proposition 6.3. Let $\mathcal{A}$ be a Grothendieck category. Let $\mathcal{U}$ be the smallest aisle in $\mathbf{D}(\mathcal{A})$ that contains an object $E$. If $E$ is compact and exceptional, then it belongs to the heart $\mathcal{C}=\mathcal{U}^{\perp}[1] \cap \mathcal{U}$ of the $t$-structure $\left(\mathcal{U}, \mathcal{U}^{\perp}[1]\right)$. The category $\mathcal{C}$ is abelian, cocomplete and $E$, as an object of $\mathcal{C}$, is small, projective and a generator. 
Proof. First, $E$ belongs to $\mathcal{U}$ and to $\mathcal{U}^{\perp}[1]$ because it is exceptional; therefore, $E \in \mathcal{C}$. The category $\mathcal{C}$ is abelian by $[\mathrm{BBD}$, Théorème 1.3.6]. To see that it is cocomplete it is enough to see that it has coproducts. This is true for $\mathcal{U}$ and $\mathcal{U}^{\perp}[1]$ by Proposition A.2 and, therefore, also for $\mathcal{C}$.

Now take any object $Y$ in $\mathcal{C}$. It belongs to $\mathcal{U}$ by definition. If we also have that $\operatorname{Hom}_{\mathcal{C}}(E, Y)=\operatorname{Hom}_{\mathbf{D}(\mathcal{A})}(E, Y)=0$, then it already belongs to $\mathcal{U}^{\perp}$ and therefore, to $\mathcal{U} \cap \mathcal{U}^{\perp}$; so $Y=0$. This shows that $E$ is a generator in $\mathcal{C}$ (in the sense of abelian categories).

Let $0 \rightarrow X \rightarrow Y \rightarrow Z \rightarrow 0$ be an exact sequence in $\mathcal{C}$. It comes from a triangle in $\mathbf{D}(\mathcal{A})$, namely, $X \longrightarrow Y \longrightarrow Z \stackrel{+}{\longrightarrow}$. Since $Z \in \mathcal{U}^{\perp}[1]$, we have that $Z[-1] \in \mathcal{U}^{\perp}$. But then $\operatorname{Hom}_{\mathbf{D}(\mathcal{A})}(E, Z[-1])=0$. The complex $E$ is exceptional; so by Lemma 6.1 . $\operatorname{Hom}_{\mathbf{D}(\mathcal{A})}(E, X[1])=0$ because $X$ belongs to $\mathcal{U}$. Taking these facts into account, we apply $\operatorname{Hom}_{\mathcal{C}}(E,-)=\operatorname{Hom}_{\mathbf{D}(\mathcal{A})}(E,-)$ to the triangle and obtain an exact sequence

$$
0 \rightarrow \operatorname{Hom}_{\mathcal{C}}(E, X) \longrightarrow \operatorname{Hom}_{\mathcal{C}}(E, Y) \longrightarrow \operatorname{Hom}_{\mathcal{C}}(E, Z) \rightarrow 0
$$

therefore, $E$ is a projective object of $\mathcal{C}$. The object $E$ is compact in $\mathbf{D}(\mathcal{A})$. Thus it is small in $\mathcal{C}$ because the functor $\operatorname{Hom}_{\mathcal{C}}(E,-)$ commutes with coproducts.

For the next results, we need some notation. Let $\mathbf{T}$ be a triangulated category and $\mathcal{U}$ an aisle in $\mathbf{T}$. The bounded part of $\mathbf{T}$ with respect to $\mathcal{U}$ is the full subcategory defined by

$$
\mathbf{T}_{\mathcal{U}}^{\mathrm{b}}:=\bigcup_{a, b \in \mathbb{Z}} \mathcal{U}[a] \cap \mathcal{U}^{\perp}[b] .
$$

The following lemma is similar to [BBD Proposition 3.1.16].

Lemma 6.4. Let $\left(\mathcal{U}, \mathcal{U}^{\perp}[1]\right)$ be a t-structure in $\mathbf{D}(\mathcal{A})$ and $\mathcal{C}$ its heart. The functor real : $\mathbf{D}^{\mathrm{b}}(\mathcal{C}) \rightarrow \mathbf{D}(\mathcal{A})_{\mathcal{U}}^{\mathrm{b}}$, defined in $[\mathrm{BBD}, 3.1 .10]$, is an equivalence of categories if, and only if, for all objects $A$ and $B$ in $\mathcal{C}, n>0$ and $f \in \operatorname{Hom}_{\mathbf{D}(\mathcal{A})}(A, B[n])$, there is an epimorphism $A^{\prime} \rightarrow A$ in $\mathcal{C}$ that erases $f$.

Proof. To check that real is fully faithful, we modify slightly the first part of the proof in loc. cit. The functor real induces a map

$$
\operatorname{Hom}_{\mathbf{D}^{\mathrm{b}}(\mathcal{C})}(A, B[n]) \rightarrow \operatorname{Hom}_{\mathbf{D}(\mathcal{A})}(A, B[n])
$$

that is an isomorphism for $n=0$. For $n>0$, the functors $\operatorname{Hom}_{\mathbf{D}^{\mathrm{b}}(\mathcal{C})}(-, B[n])$ are Yoneda's Ext in $\mathcal{C}$, therefore making part of a (contravariant) universal $\delta$-functor. The same is true for $\operatorname{Hom}_{\mathbf{D}(\mathcal{A})}(-, B[n])$, because the conditions of Grothendieck's characterization of universal $\delta$-functors $([\mathrm{Gr}$ Proposition 2.2.1]) hold by hypothesis.

The essential image of real is $\mathbf{D}(\mathcal{A})_{\mathcal{U}}^{\mathrm{b}}$ by the same argument as in BBD, Proposition 3.1.16].

For convenience, for a separated divisorial scheme $X$, let us say that a tilting complex of quasi-coherent sheaves $\mathcal{E}$ is special if the aisle generated by $\mathcal{E}$ in $\mathbf{D}(\mathrm{Q} \operatorname{co}(X))$ is rigid.

Proposition 6.5. Let the category $\mathcal{A}$ be either $R$-Mod for an arbitrary ring $R$ or $\mathrm{Qco}(X)$ for a separated divisorial scheme $X$. Let $E$ be a tilting object of $\mathbf{D}(\mathcal{A})$, which we suppose special in the scheme case. Let $\mathcal{C}$ be the heart of the t-structure that $E$ defines in $\mathbf{D}(\mathcal{A})$. Then, $\mathbf{D}^{\mathrm{b}}(\mathcal{C})$ is equivalent to $\mathbf{D}^{\mathrm{b}}(\mathcal{A})$. 
Proof. Let $\mathcal{U}$ denote the aisle determined by $E$. The object $E \in \mathbf{D}^{-}(\mathcal{A})$, because it is bounded (Lemma 4.3 and Lemma [5.5). Therefore, we have that $\mathcal{U}[a] \subset \mathbf{D}^{-}(\mathcal{A})$ for every $a \in \mathbb{Z}$. Using Proposition 4.5 for $\mathcal{A}=R$-Mod and Proposition [5.6 for $\mathcal{A}=\mathrm{Qco}(X)$ we see that $\mathcal{U}^{\perp}[b] \subset \mathbf{D}^{+}(\mathcal{A})$ for every $b \in \mathbb{Z}$. As a consequence $\mathbf{D}(\mathcal{A})_{\mathcal{U}}^{\mathrm{b}} \subset \mathbf{D}^{\mathrm{b}}(\mathcal{A})$.

Let us show that $\mathbf{D}(\mathcal{A})_{\mathcal{U}}^{\mathrm{b}}=\mathbf{D}^{\mathrm{b}}(\mathcal{A})$. Given $M \in \mathbf{D}^{\mathrm{b}}(\mathcal{A})$, we will find $a, b \in \mathbb{Z}$ such that $M \in \mathcal{U}[a]$ and $M \in \mathcal{U}^{\perp}[b]$. Let us check first that $\mathbf{R H o m}(E, M)$ is a bounded complex. Indeed, in the case that $\mathcal{A}=R$-Mod the compact object $E$ can be represented by a bounded complex of projective modules (Lemma 4.3) and, therefore, $\mathbf{R H o m}^{\prime}(E, M) \sim \operatorname{Hom}^{\cdot}(E, M)$ which is clearly bounded. In the case $\mathcal{A}=\mathrm{Q} \operatorname{co}(X)$, the compact complex $E$ can be represented by a bounded complex of locally free finite-type modules (Lemma 5.5) and we have that $\mathbf{R} \mathcal{H}_{\text {om }}{ }^{\prime}(E, M) \sim \mathcal{\rightarrow} \mathcal{H} \circ m^{\prime}(E, M)$, because locally free modules are acyclic for $\mathcal{H o m}^{\cdot}(-, M)$ from which it follows that $\mathbf{R} \mathcal{H o m}^{\cdot}(E, M)$ is bounded. Now, $X$ being separated, the derived functor $\mathbf{R} \Gamma(X,-)$ can be computed via Čech resolutions ([EGA III 1.4.1]) and these resolutions are bounded because $X$ is quasi-compact. Therefore, $\mathbf{R} \Gamma(X,-)$ takes bounded complexes in $\mathcal{A}$ to bounded complexes of abelian groups and

$$
\mathbf{R H o m}^{\prime}(E, M) \cong \mathbf{R} \Gamma\left(X, \mathbf{R} \mathcal{H}_{\text {om }}(E, M)\right)
$$

is a bounded complex as claimed. Then there exist $a, b \in \mathbb{Z}$ such that

$$
\operatorname{Hom}_{\mathbf{D}(\mathcal{A})}(E[j], M)=H^{-j} \mathbf{R} \operatorname{Hom}(E, M)=0
$$

for all $j \geq b$ and $j<a$.

Note that $\mathcal{U}^{\perp}[b]=\mathcal{U}[b]^{\perp}$ and the aisle $\mathcal{U}[b]$ is generated by $E[b]$. We apply Lemma 3.1 and conclude that $M \in \mathcal{U}^{\perp}[b]$ because $\operatorname{Hom}_{\mathbf{D}(\mathcal{A})}(E[j], M)=0$ for all $j \geq b$.

To see that $M \in \mathcal{U}[a]$, we prove that in the distinguished triangle

$$
\tau_{\mathcal{U}}^{\leq-a} M \longrightarrow M \longrightarrow \tau_{\mathcal{U}}^{\geq 1-a} M \stackrel{+}{\longrightarrow},
$$

the object $B:=\tau_{\mathcal{U}}^{\geq 1-a} M$ is 0 . The aisle $\mathcal{U}[a]$ is generated by $E[a]$, that is, a tilting complex, and then

$$
\operatorname{Hom}_{\mathbf{D}(\mathcal{A})}(E[a+j], E[a])=\operatorname{Hom}_{\mathbf{D}(\mathcal{A})}(E[j], E)=0,
$$

for $j<0$. By Lemma 6.1 if $N \in \mathcal{U}[a]$ we have that $\operatorname{Hom}_{\mathbf{D}(\mathcal{A})}(E[a+j], N)=0$ for all $j<0$. Also, $\operatorname{Hom}_{\mathbf{D}(\mathcal{A})}(E[a+j], M)=0$ for all $j<0$. Applying the cohomological functor $\operatorname{Hom}_{\mathbf{D}(\mathcal{A})}(E,-)$ to the distinguished triangle above we obtain an exact sequence from which it follows that $\operatorname{Hom}_{\mathbf{D}(\mathcal{A})}(E[a+j], B)=0$ for $j<0$. On the other hand, we have that $\operatorname{Hom}_{\mathbf{D}(\mathcal{A})}(E[a+j], B)=0$ for $j \geq 0$ because $B \in \mathcal{U}[a]^{\perp}$. Summing up, since $E$ is a generator, $B=0$.

Finally, consider the functor real : $\mathbf{D}^{\mathrm{b}}(\mathcal{C}) \rightarrow \mathbf{D}(\mathcal{A})_{\mathcal{U}}^{\mathrm{b}}$. To see that it is an equivalence, it is only left to check that the hypothesis of the previous lemma holds. Let $A$ and $B$ in $\mathcal{C}, n>0$ and $f \in \operatorname{Hom}(A, B[n])$. By Proposition [6.3 $E$ is projective and a generator in $\mathcal{C}$. We can take an epimorphism $A^{\prime} \rightarrow A$, where $A^{\prime}$ is a direct sum of copies of $E$, and this epimorphism erases $f$, as required.

Theorem 6.6. Let $R$ and $S$ be rings. We have an equivalence:

(i) The categories $\mathbf{D}^{\mathrm{b}}(S)$ and $\mathbf{D}^{\mathrm{b}}(R)$ are equivalent.

(ii) There is a tilting object $E$ in $\mathbf{D}(R)$, such that $\operatorname{Hom}_{\mathbf{D}(R)}(E, E)=S$. 
Proof. $(i) \Rightarrow($ ii $)$ The object of $\mathbf{D}^{\mathrm{b}}(R)$ corresponding to $S$ by the equivalence is clearly tilting.

$($ ii $) \Rightarrow($ i) Consider the $t$-structure generated by the tilting object $E$ in $\mathbf{D}(R)$. Let $\mathcal{C}$ be its heart. By Proposition 6.5, $\mathbf{D}^{\mathrm{b}}(R)$ is equivalent to $\mathbf{D}^{\mathrm{b}}(\mathcal{C})$. Also, by Proposition 6.3 the object $E$ belongs to $\mathcal{C}$ and it is a small projective generator. But an abelian category with a small projective generator is a module category by [M] Chap. IV, Theorem 4.1 p. 104]. Then the ring $S:=\operatorname{Hom}_{\mathcal{C}}(E, E)$ is such that $S$-Mod is equivalent to $\mathcal{C}$ and, consequently, $\mathbf{D}^{\mathrm{b}}(S)$ is equivalent to $\mathbf{D}^{\mathrm{b}}(R)$.

Remark. The previous theorem gives another proof of $(\mathrm{b}) \Leftrightarrow(\mathrm{e})$ in $[\mathrm{R}$, Theorem 6.4]. We have to say that Rickard was also inspired in the construction given in [BBD] to get a totalization of bicomplexes "up to homotopy". The difference is our explicit use of the functor real. This shows that constructing a $t$-structure is a general means for establishing an equivalence.

We also point out that some technicalities from Sections 4 and 5 could be avoided if we had an analogue of the functor real for unbounded derived categories. This construction would be feasible once we had a satisfactory theory of totalization for unbounded bicomplexes which, at present, does not seem possible to obtain.

Theorem 6.7. Let $X$ be a separated divisorial scheme. If there is a special tilting object $\mathcal{E}$ in $\mathbf{D}(\mathrm{Q} \operatorname{co}(X))$, then the ring $R=\operatorname{Hom}_{\mathbf{D}(Q \operatorname{co}(X))}(\mathcal{E}, \mathcal{E})$ is such that $\mathbf{D}^{\mathrm{b}}(\mathrm{Q} \operatorname{co}(X))$ is equivalent to $\mathbf{D}^{\mathrm{b}}(R)$.

Proof. It follows from Proposition 6.5, again using the characterization of module categories in $[\mathrm{M}]$ as in the previous theorem.

6.8. We see now how Bellinson's equivalence can be interpreted under the light of the previous results. Let $\mathbf{P}_{K}^{d}$ be the $d$-dimensional projective space over a field $K$. Denote by $\mathcal{O}(1)$ the canonical ample invertible sheaf and by $\Omega_{\mathbf{P}}^{1}$ the sheaf of differential forms. Consider the families of locally free sheaves $\mathcal{S}_{\Lambda}:=$ $\left\{\mathcal{O}_{\mathbf{P}}, \Omega_{\mathbf{P}}^{1}(1), \ldots, \Omega_{\mathbf{P}}^{d}(d)\right\}$ and $\mathcal{S}_{S}:=\left\{\mathcal{O}_{\mathbf{P}}, \mathcal{O}_{\mathbf{P}}(-1), \ldots, \mathcal{O}_{\mathbf{P}}(-d)\right\}$. They are obviously made of compact objects in $\mathbf{D}\left(\mathrm{Qco}\left(\mathbf{P}_{K}^{d}\right)\right)$. From [B1, Lemma 2], the objects

$$
\begin{aligned}
& \mathcal{E}_{\Lambda}:=\mathcal{O}_{\mathbf{P}} \oplus \Omega_{\mathbf{P}}^{1}(1) \oplus \cdots \oplus \Omega_{\mathbf{P}}^{d}(d), \\
& \mathcal{E}_{S}:=\mathcal{O}_{\mathbf{P}} \oplus \mathcal{O}_{\mathbf{P}}(-1) \oplus \cdots \oplus \mathcal{O}_{\mathbf{P}}(-d)
\end{aligned}
$$

are exceptional. Also, from the proof of [B1, Theorem, p. 215] they are generators of $\mathbf{D}\left(\mathrm{Qco}\left(\mathbf{P}_{K}^{d}\right)\right)$. Let

$$
\begin{aligned}
& R_{\Lambda}:=\operatorname{End}_{\mathbf{D}\left(\mathrm{Qco}\left(\mathbf{P}_{K}^{d}\right)\right)}\left(\mathcal{E}_{\Lambda}\right), \\
& R_{S}:=\operatorname{End}_{\mathbf{D}\left(\mathbf{Q c o}\left(\mathbf{P}_{K}^{d}\right)\right)}\left(\mathcal{E}_{S}\right) .
\end{aligned}
$$

Denote simply by $R$ either $R_{\Lambda}$ or $R_{S}$, and, analogously, let $\mathcal{E}$ be either $\mathcal{E}_{\Lambda}$ or $\mathcal{E}_{S}$, respectively. For a triangulated category $\mathbf{T}$, denote by $\mathbf{T}_{\mathrm{cp}}$ the full subcategory of compact objects in $\mathbf{T}$. We have the following.

Corollary 6.9. There is an equivalence between the categories $\mathbf{D}^{\mathrm{b}}\left(\mathrm{Q} \operatorname{co}\left(\mathbf{P}_{K}^{d}\right)\right)$ and $\mathbf{D}^{\mathrm{b}}(R)$. Moreover, this equivalence induces one between $\mathbf{D}_{\mathrm{c}}^{\mathrm{b}}\left(\mathrm{Qco}\left(\mathbf{P}_{K}^{d}\right)\right)$ and $\mathbf{D}(R)_{\mathrm{cp}}$.

Proof. In view of Theorem 6.7, the only thing left to check is that $\mathcal{E}$ is special in either case; in other words, that the aisle $\mathcal{U}$ generated by $\mathcal{E}$ (or equivalently, by the families $\mathcal{S}_{\Lambda}$ or $\mathcal{S}_{S}$ ) is rigid. 
The key argument in Berllinson's papers is that one can express every coherent sheaf $\mathcal{F}$ over $\mathbf{P}_{K}^{d}$ as the 0th homology of a complex concentrated in degrees from $-d$ to $d$ (denoted $L_{\Lambda}$ and $L_{S}$ in [B2, Lemma 3]) whose components are formed by coproducts of the objects in the families $\mathcal{S}_{\Lambda}$ and $\mathcal{S}_{S}$, respectively. These resolutions are called the Beilinson monads of the sheaf $\mathcal{F}$. It follows that $\mathcal{F}$ can be obtained by a process of producing mapping cones, coproducts and positive translations up to $d$ of objects in the families and therefore $\mathcal{F} \in \mathcal{U}[-d]$.

It follows that, for $k \ll 0$ and $i \in\{0, \ldots, d\}$, the objects $\Omega_{\mathbf{P}}^{i}(i+k)$, twists of the generators of the aisle $\mathcal{U}_{\Lambda}$, belong to $\mathcal{U}_{\Lambda}[-d]$; and analogously for the sheaves $\mathcal{O}(-i+k)$ and the aisle $\mathcal{U}_{S}$. Now it is easy to see that, once this fact holds for the generators of an aisle $\mathcal{U}$, it holds also for all the objects in $\mathcal{U}$. Therefore, we can apply Proposition 5.1 to $\mathcal{U}_{\Lambda}$ or $\mathcal{U}_{S}$, respectively, and conclude that they are both rigid.

Finally, the object $\mathcal{E}$ is projective and a generator viewed as an $R$-module, and, therefore, is a compact generator of $\mathbf{D}(R)$. By $\left[\mathrm{N}\right.$, Lemma 2.2] $\mathbf{D}(R)_{\mathrm{cp}}$ is the smallest thick subcategory of $\mathbf{D}^{\mathrm{b}}(R)$ that contains $\mathcal{E}$. The functor real extends the inclusion of the heart to a map between derived categories; so it takes $\mathcal{E}$ to itself. But $\mathcal{E}$ is also a compact generator of $\mathbf{D}\left(\mathrm{Q} \operatorname{co}\left(\mathbf{P}_{K}^{d}\right)\right)$, and by the cited lemma, the smallest thick subcategory of $\mathbf{D}^{\mathrm{b}}\left(\mathrm{Q} \operatorname{co}\left(\mathbf{P}_{K}^{d}\right)\right)$ containing $\mathcal{E}$ is $\mathbf{D}\left(\mathrm{Q} \operatorname{co}\left(\mathbf{P}_{K}^{d}\right)\right)_{\mathrm{cp}}$. The functor real preserves thick subcategories and therefore both are equivalent. Finally, $\mathbf{D}_{\mathrm{c}}^{\mathrm{b}}\left(\mathrm{Q} \operatorname{co}\left(\mathbf{P}_{K}^{d}\right)\right)=\mathbf{D}\left(\mathbf{Q} \operatorname{co}\left(\mathbf{P}_{K}^{d}\right)\right)_{\mathrm{cp}}$ by the Auslander-Buchsbaum-Serre theorem, because $\mathbf{P}_{K}^{d}$ is a regular scheme.

\section{Appendix: Compactly generated $t$-STRuCtures}

In this section we will give a solution to the Problem in Section 1 for general triangulated categories under the hypothesis that the generating objects for the complete pre-aisle are compact. This gives a criterion for the existence of $t$ structures applicable in very general settings. Also, the good behavior of these kinds of objects will allow us to get information about the $t$-structure already in the case of a derived category, as treated in the main text.

Theorem A.1. Let $\mathcal{S}=\left\{E_{\alpha} / \alpha \in A\right\}$ be a set of compact objects in a triangulated category $\mathbf{T}$. Let $\mathcal{U}$ be the smallest cocomplete pre-aisle of $\mathbf{T}$ that contains the family $\mathcal{S}$. Then $\mathcal{U}$ is an aisle in $\mathbf{T}$.

Proof. This argument is inspired in [N Lemma 1.7, p. 554]. Letting $M \in \mathbf{T}$, let us show how to construct a distinguished triangle $N \rightarrow M \rightarrow B \stackrel{+}{\rightarrow}$ with $N \in \mathcal{U}$ and $B \in \mathcal{U}^{\perp}$.

This time ordinary induction will do. Let $B_{0}:=M$. Suppose $B_{n-1}$ is already constructed. Let $\Omega_{n-1}:=\bigcup_{\alpha \in A, k \in \mathbb{N}} \operatorname{Hom}_{\mathbf{C}(R)}\left(E_{\alpha}[k], B_{n-1}\right)$ and define $B_{n}$ by the triangle

$$
\bigoplus \bigoplus_{\Omega_{n-1}} E_{\alpha}[k] \stackrel{\rho}{\longrightarrow} B_{n-1} \stackrel{\mu}{\longrightarrow} B_{n} \stackrel{+}{\longrightarrow},
$$

where $\rho$ is defined by the universal property of the coproduct, as in Proposition 3.2. Let $B=\underset{n \in \mathbb{N}}{\operatorname{holim}} B_{n}$ which, by the remark after Lemma 2.1] may be defined 
by the distinguished triangle

$$
\bigoplus_{n \in \mathbb{N}} B_{n} \stackrel{1-\mu}{\longrightarrow} \bigoplus_{n \in \mathbb{N}} B_{n} \longrightarrow B \stackrel{+}{\longrightarrow} .
$$

The object $B$ belongs to $\mathcal{U}^{\perp}$. Indeed, using Lemma 3.1, it is enough to check that for any $\alpha \in A$ and $k \geq 0, \operatorname{Hom}_{\mathbf{T}}\left(E_{\alpha}[k], B\right)=0$. But, applying the functor $\operatorname{Hom}_{\mathbf{T}}\left(E_{\alpha}[k],-\right)$ to the previous triangle,

$$
\operatorname{Hom}_{\mathbf{T}}\left(E_{\alpha}[k], \operatorname{holim}_{n \in \mathbb{N}} B_{n}\right) \cong \lim _{n \in \mathbb{N}} \operatorname{Hom}_{\mathbf{T}}\left(E_{\alpha}[k], B_{n}\right) .
$$

But this limit is 0 because the image of the map

$$
\operatorname{Hom}_{\mathbf{T}}\left(E_{\alpha}[k], B_{n-1}\right) \rightarrow \operatorname{Hom}_{\mathbf{T}}\left(E_{\alpha}[k], B_{n}\right)
$$

is formed by maps that factor through $\mu$ in triangle (A.1) and two successive maps in a triangle are 0 , whence the claim.

The object $N$ belongs to $\mathcal{U}$ copying the argument in Proposition 3.2 .

Remark. Observe that we cannot simplify and take $\bigoplus E_{i}$ as the generator of the aisle because this object does not need to be compact when the family is not finite.

Proposition A.2. Keeping the notation as in the previous theorem, the associated functors $\tau_{\mathcal{U}}^{\geq n}, \tau_{\mathcal{U}}^{\leq n}: \mathbf{T} \rightarrow \mathbf{T}$ preserve coproducts with $n \in \mathbb{Z}$.

Proof. It is obviously enough to treat the case $\tau_{\overline{\mathcal{U}}}^{\geq 1}$. But then it is enough to see that its essential image, $\mathcal{U}^{\perp}$, is closed under the formation of coproducts in $\mathbf{T}$. Let $\left\{G_{i} / i \in I\right\}$ be a family of objects in $\mathcal{U}^{\perp}$. By using Lemma 3.1 once again, we have to check that

$$
\operatorname{Hom}_{\mathbf{T}}\left(E_{\alpha}[k], \bigoplus_{i \in I} G_{i}\right)=0
$$

for every $\alpha \in A$ and $k \geq 0$. But each $E_{\alpha}[k]$ is compact and, therefore,

$$
\operatorname{Hom}_{\mathbf{T}}\left(E_{\alpha}[k], \bigoplus_{i \in I} G_{i}\right)=\bigoplus_{i \in I} \operatorname{Hom}_{\mathbf{T}}\left(E_{\alpha}[k], G_{i}\right)=0
$$

as wanted.

Remark. This result is an adaptation of Neeman-Ravenel's argument for compactly generated localizing subcategories to aisles (see [N] Proposition 1.9]).

\section{REFERENCES}

[AJL] Alonso Tarrío, L.; Jeremías López, A.; Lipman, J.: Local homology and cohomology on schemes, Ann. Scient. École Norm. Sup. 30 (1997), 1-39. MR 98d:14028

[AJS] Alonso Tarrío, L.; Jeremías López, A.; Souto Salorio, Ma. José: Localization in categories of complexes and unbounded resolutions, Canad. J. Math. 52 (2000), no. 2, 225-247. MR 2001i:18019]

[B1] Beylinson, A. A.: Coherent sheaves on $\mathbf{P}^{n}$ and problems in linear algebra. Functional Anal. Appl. 12 (1978), no. 3, 214-216 (1979).

[B2] : The derived category of coherent sheaves on $\mathbf{P}^{n}$. Selected translations. Selecta Math. Soviet. 3 (1983/84), no. 3, 233-237. MR 88h:14021

[BBD] Beŭlinson, A. A.; Bernstein, J.; Deligne, P.: Faisceaux pervers. Analysis and topology on singular spaces, I (Luminy, 1981), 5-171, Astérisque, 100, Soc. Math. France, Paris, 1982. MR 86g:32015

[BN] Bökstedt, M.; Neeman, A.: Homotopy limits in triangulated categories, Compositio Math. 86 (1993), 209-234. MR 94f:18008 
[GP] Popesco, N.; Gabriel, P.: Caractérisation des catégories abéliennes avec générateurs et limites inductives exactes. C. R. Acad. Sci. Paris 258 (1964), 4188-4190. MR 29:3518

[Gr] Grothendieck, A.: Sur quelques points d'algèbre homologique. Tôhoku Math. J. (2) 9 (1957), 119-221. MR 21:1328

[EGA I] Grothendieck, A.; Dieudonné, J. A.: Eléments de Géométrie Algébrique I, Grundlehren der math. Wiss. 166, Springer-Verlag, Heidelberg, 1971.

[EGA III] _Eléments de Géométrie Algébrique III, Étude cohomologique des faisceaux cohérents. I. Inst. Hautes Etudes Sci. Publ. Math. 11 (1961). MR 36:177c

[KV1] Keller, B. and Vossieck, D.: Sous les catégories dérivées, C. R. Acad. Sci. Paris Sér. I Math. 305 (1987), no. 6, 225-228. MR 88m:18014

[KV2] _ Aisles in derived categories, Bull. Soc. Math. Belg. Sér. A 40 (1988), no. 2, 239-253. MR 90j:16047

[I] Illusie, L.: Existence de résolutions globales, in Théorie des Intersections et Théorème de Riemann-Roch (SGA 6), Lecture Notes in Math., no. 225, Springer-Verlag, New York, 1971, pp. 160-222

[L] Lévy, A.: Basic set theory. Springer-Verlag, Berlin-New York, 1979. MR 80k:04001

[M] Mitchell, B.: Theory of categories, Academic Press, New York, 1965. MR 34:2647

[N] Neeman, A.: The connection between the $K$-theory localization theorem of Thomason, Trobaugh and Yao and the smashing subcategories of Bousfield and Ravenel, Ann. Sci. École Norm. Sup. (4) 25 (1992), no. 5, 547-566. MR 93k:18015

[R] Rickard, J.: Morita theory for derived categories, J. London Math. Soc. (2) 39 (1989), no. 3, 436-456. MR 91b:18012

[St] Stenström, B.: Rings of quotients. An introduction to methods of ring theory. Grund. der math. Wiss. Bd. 217. Springer-Verlag, Berlin-Heidelberg-New York, 1975. MR 52:10782

[TT] Thomason, R. W.; Trobaugh, T.: Higher algebraic $K$-theory of schemes and of derived categories. The Grothendieck Festschrift, Vol. III, 247-435, Progr. Math., 88, Birkhäuser Boston, Boston, MA, 1990. MR 92f:19001

[V] Verdier, J.-L.: Categories dérivées. Quelques résultats (Etat 0). Semin. Geom. algebr. Bois-Marie, SGA 4 $\frac{1}{2}$, Lect. Notes Math. 569, 262-311 (1977).

Departamento de Álxebra, Facultade de Matemáticas, Universidade de Santiago de Compostela, E-15782 Santiago de Compostela, Spain

E-mail address: leoalonso@usc.es

Departamento de Álxebra, Facultade de Matemáticas, Universidade de Santiago de Compostela, E-15782 Santiago de Compostela, Spain

E-mail address: jeremias@usc.es

Facultade de Informática, Campus de Elviña, Universidade da Coruña, E-15071 A CORUÑA, Spain

E-mail address: mariaj@udc.es 